\title{
Direct numerical simulation of vortex ring evolution from the laminar to the early turbulent regime
}

\author{
P. J. ARCHER, T. G. THOMAS AND G. N. COLEMAN \\ University of Southampton - Aerospace Engineering, Highfield Campus, \\ University Road Southampton, SO17 1BJ, UK \\ pja@soton.ac.uk
}

(Received 19 October 2006 and in revised form 19 October 2007)

\begin{abstract}
Direct numerical simulation is used to study the temporal development of single vortex rings at various Reynolds numbers and core thicknesses. Qualitative differences between the evolution of thin- and thick-core rings are observed leading to a correction factor to the classical equation for the ring translational velocity. We compare the obtained linear modal growth rates with previous work, highlighting the role of the wake in triply periodic numerical simulations. The transition from a laminar to a turbulent ring is marked by the rearrangement of the outer core vorticity into a clearly defined secondary structure. The onset of the fully turbulent state is associated with shedding of the structure in a series of hairpin vortices. A Lagrangian particle analysis was performed to determine the ring entrainment and detrainment properties and to investigate the possibility of an axial flow being generated around the circumference of the core region prior to the onset of turbulence.
\end{abstract}

\section{Introduction}

Vortex rings are interesting for a number of reasons, ranging from their ubiquitous nature to the fact that their growth, instability and breakdown represent a prototypical turbulent flow. We focus here upon the behaviour of a single ring during this breakdown process, as it transitions from a laminar state via linear and nonlinear processes into the early stages of the fully turbulent regime.

The instability of vortex rings was first studied by Krutzsch (1939) who showed that a ring is unstable, and develops stationary azimuthal waves around its circumference prior to becoming turbulent. However, it was not until the investigation of Crow (1970) into aircraft wakes that the full process of vortex-ring instability, growth and transition was addressed. Maxworthy (1972, 1974, 1977), and Widnall \& Sullivan (1973) then confirmed experimentally that during the laminar phase, stationary azimuthal waves grow in the conical surface at $45^{\circ}$ to the axis of ring-propagation, and that their wavenumber depends on the slenderness ratio $\epsilon$ of core radius $\delta$ to ring radius $R$. Widnall \& Tsai (1977) then proved that the disturbances are unstable for rings with a uniform distribution of vorticity in their core, and found an expression for the growth rate of the most amplified wave in the limit of very thin cores (3.3). Shariff, Verzicco \& Orlandi (1994) developed a viscous correction to this growth rate, based on their direct numerical simulations (DNS) of single rings for a range of $\epsilon$. This correction has been called into question by the experiments of Dazin, Dupont \& Stanislas (2006a), who suggest that it is too small because amongst other things the 
Shariff et al. prediction is only valid for purely Gaussian vortex cores. However, this takes no account of the fact that in the DNS the initially Gaussian distribution quickly equilibrates to a skewed Gaussian-like profile similar to that of the experiments (see $\S 3.2$ ). One of the aims of this study is to resolve this issue. The vortex ring instability is an example of the elliptical instability, a review of which can be found in Kerswell (2002).

Another aim is to better understand the nonlinear growth and breakdown of the vortex ring instability. Following the linear phase is a short period of nonlinear wave growth, culminating in a turbulent vortex ring. Maxworthy (1977) describes the final stages of the laminar ring as rotation and preferential breaking of the instability waves resulting in a net ('swirling') flow in the form of a solitary wave propagating along the axis of the ring circumference. The notion of an axial flow is supported by Shariff et al. (1994) and by the experiments of Naitoh et al. (2002) (following Maxworthy, the term 'axial' will be used to indicate the circumferential axis of the vortex core). However, both these studies show that the flow is in fact initiated prior to the breaking of the instability waves. Dazin et al. (2006a,b) used particle image velocimetry (PIV) and novel flow visualizations to investigate the linear and nonlinear phases. They showed that the nonlinear phase was heralded by the rapid growth of higher-order harmonics of the most unstable linear modes. This is followed by the rapid growth of the $n=0$ mode in the azimuthal energy component corresponding to a mean axial flow (consistent with Shariff et al. 1994), accompanied by the development of vortical structures on the (outer) periphery of the ring, leading to ejection of vorticity into the wake. Vortical structures then develop in the interior of the ring immediately before the breakdown to turbulence, causing the authors to propose that the structures are progressively wrapped around the core; this is consistent with the vortex tubes observed in the experiments of Schneider (1980) during the latter stages of transition. Bergdorf, Koumoutsakos \& Leonard (2007) investigated the vortical structures numerically, suggesting that they originate from locally stretched regions of the deformed core. Their ring parameters were chosen to match the experiments of Wiegand \& Gharib (1994), which tracked vortex rings at an initial Reynolds number (based on ring circulation $\Gamma$ ) of 7500 through the laminar into the turbulent regime. Qualitative agreement was found between the two studies which showed that the turbulent ring sheds vortical structure into the wake in a series of hairpin vortices. The loss of discrete structures leads to a 'staircase-like' decay in time of circulation and velocity, with the velocity lagging the circulation by a small amount. Glezer \& Coles (1990) also noted peripheral vortex structures in experimentally generated turbulent rings, inferring that they are likely to be vortex tubes of alternating sign wrapped around the main core, with their presence influencing the local entrainment and detrainment dynamics.

A key feature of a vortex ring is that the core is surrounded by a co-moving mass of fluid, known as the entrainment bubble. The size of the entrainment bubble is not fixed and its rate of change is defined by the balance between entrainment and detrainment. Maxworthy (1972) proposed a model for diffusive entrainment whereby the irrotational fluid close to the vortex is contaminated by vorticity diffusing across the vortex bubble surface. A proportion of this fluid is then entrained through the rear of the bubble into the vortex ring, the remainder ejected into the wake. Powerlaw ratios were given for the decay of both the overall ring velocity and circulation, but these power laws were found not to be universal by Dabiri \& Gharib (2004), who considered rings propagating into both an ambient fluid and an oncoming flow of uniform velocity. They were, however, able to confirm part of Maxworthy's 
entrainment model, showing that vorticity is diffused across the vortex bubble and is present in the wake.

The ability of a ring to directly entrain fluid has also been studied by Glezer \& Coles (1990), who used a streamfunction approach in similarity coordinates to predict net entrainment of particles for the case of a fully turbulent ring. The concept of fluid entrainment and detrainment has been further refined by the application of stable and unstable manifolds, taken from dynamical systems theory, to the vortex ring boundary. In a two-dimensional numerical study, Rom-Kedar, Leonard \& Wiggins (1990) perturbed a vortex pair with a time-periodic strain field, which was found to induce lobes of entrainment and detrainment. Shariff, Leonard \& Ferziger (2006) then showed numerically that some typical features of turbulent rings, such as the entrainment of particles placed in the path of the vortex ring and striations in the ring wake, could be explained by the behaviour of the unstable manifolds associated with an axisymmetric vortex ring with a rotating elliptic core. Shadden, Dabiri \& Marsden (2006) demonstrated that the dynamical systems analysis could be extended to aperiodic flows using finite-time Lyapunov fields to identify Lagrangian coherent structures. They analysed piston-generated vortex rings and found that lobe dynamics developed that were consistent with regions of entrainment and detrainment. The development of lobes suggests splitting of the heteroclinic connection between the front and rear stagnation points which require perturbations to the idealized steady-state vortex-ring structure. These perturbations are evident in the experiments of Kumar, Arakeri \& Shankar (1995), of rings at similar Reynolds numbers to Shadden et al. By taking high-resolution temporal measurements, they found that in the frame of reference attached to the ring, the translational velocity of laminar rings oscillates in time. The oscillation was of greatest amplitude immediately after the ring formation and reduced with time until it was within experimental error.

In this paper, we present results from DNS of single naturally evolving laminar vortex rings with different relative core thicknesses and Reynolds number, and examine their initial breakdown and transition into the early stages of turbulence. The aim is to extend the nonlinear analysis begun by Shariff et al. (1994). After the mathematical and numerical preliminaries are presented in $\S 2$, the cases of interest are introduced in $\S 3.1$, along with energy-budget histories and their implications regarding the numerical fidelity of the results. We next investigate the laminar evolution of the ring (§3.2), and the wake generation, modal growth and wave breaking during the linear $(\$ 3.3)$ and nonlinear (\$3.4) regimes, with special attention paid to the generation of secondary vortical structure. Finally we investigate, using Lagrangian particlepath analysis, the relationship between the amount of entrainment or detrainment and the various stages of the ring evolution ( $\$ 3.5)$ and the possibility of an axial flow generation in the core region $(\S 3.6)$. A summary and conclusions are offered in $\S 4$.

\section{Mathematical background}

We consider a single vortex ring of radius $R$ and core radius $\delta$, with circulation $\Gamma$ and Reynolds number $R e \equiv \Gamma / \nu$, with impulse $\boldsymbol{P}$ propagating along the positive $z$-direction with respect to Cartesian coordinates $\boldsymbol{x}=(x, y, z)$ and with corresponding velocity components $\boldsymbol{u}=(u, v, w)$ (see figure 1). The vortex is initiated such that it is centred about $\boldsymbol{x}=(0,0,0)$ at time $t=0$ and, after an initial adjustment (see $\S 3.2)$, 


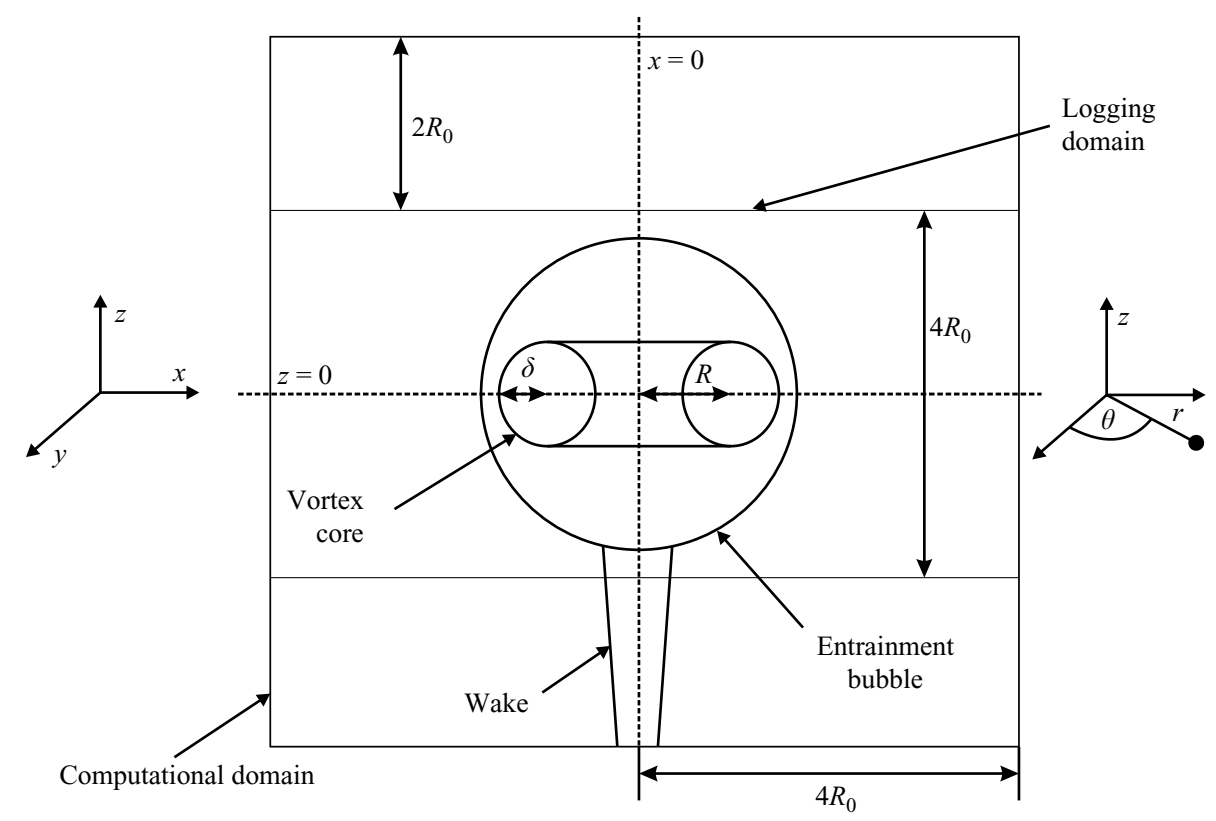

FIGURE 1. Schematic diagram of the vortex ring domain.

propagates at a velocity $U$ that depends on its instantaneous parameters according to

$$
U=\frac{\Gamma}{4 \pi R}\left[\ln \frac{8 R}{a}+C\right],
$$

where $C$ is a function of the shape of the vorticity distribution and $a$ is a measure of the core radius. For a uniform distribution $C=-1 / 4$ (Lamb 1932), and for a Gaussian core vorticity distribution $C \simeq-0.558$ (Saffman 1970). Historically, the speed-effective core radius $a_{e}$ is defined as the core radius of a vortex ring propagating at an equivalent speed and with uniform vorticity distribution. Experimentalists also often define a core measure $a_{1}$, the distance from the core centre to the point of maximum tangential velocity. For a Gaussian core $a_{e}=1.3607 \delta$ and $a_{1}=1.1214 \delta$.

We follow Saffman (1970) and define the vortex-ring geometry in terms of integral parameters. For example, under the assumption of axisymmetry (reasonable for a laminar ring), measures of the ring radius can be extracted from the first and second radial moments of the azimuthal vorticity $\omega_{\theta}$, with

$$
R_{\theta}=\frac{1}{\Gamma} \int r \omega_{\theta} \mathrm{d} r \mathrm{~d} z, \quad R_{2}^{2}=\frac{1}{\Gamma} \int r^{2} \omega_{\theta} \mathrm{d} r \mathrm{~d} z,
$$

where the circulation $\Gamma$ and impulse $P$ take their usual forms,

$$
\Gamma=\int \omega_{\theta} \mathrm{d} r \mathrm{~d} z, \quad P=\pi R_{2}^{2} \Gamma,
$$

and $r^{2}=x^{2}+y^{2}$. Equivalent three-dimensional formulae can be derived for more general non-axisymmetric distributions of vorticity. The ring radius $R_{\theta}$ can be interpreted as the radial $\omega_{\theta}$-weighted average, and the core thickness $\delta_{\theta}$ as the $\omega_{\theta}$-weighted measure of its spread. For a Gaussian $\omega_{\theta}$ distribution we have

$$
\delta_{\theta}^{2}=2\left(R_{2}^{2}-R_{\theta}^{2}\right) .
$$


These quantities are referred to below as impulse-weighted measures of the vortex geometry; they are particularly useful because of their close relationship to the integral parameters. It is also convenient to define an integral measure of the ring radius $R_{\Omega}$ based on the first moment of enstrophy via

$$
R_{\Omega}=\frac{1}{2 \Omega} \int r|\omega|^{2} \mathrm{~d} x \mathrm{~d} y \mathrm{~d} z,
$$

where $\Omega=\int|\omega|^{2} \mathrm{~d} x \mathrm{~d} y \mathrm{~d} z$ is the total enstrophy. The effect of viscosity is to diffuse the core, which for a core with a Gaussian profile is (Saffman 1970)

$$
\delta^{2}(t)=\delta_{0}^{2}+4 v t .
$$

Since the laminar ring produces a wake of shed vorticity, all characteristic ring radii and integral properties presented in this paper have been calculated over a cubic 'logging domain', the top and bottom of which are two initial ring radii $R_{0}$ from the origin (see figure 1). This prevents the wake from artificially distorting measures of the ring parameters.

\subsection{Numerical approach}

The incompressible Navier-Stokes equations are discretized on a conventional staggered grid using second-order finite differences in space and the Adams-Bashforth algorithm in time, with continuity imposed by applying pressure-correction methods with a parallel multigrid Poisson solver (Yao et al. 2001). A uniform Cartesian grid is employed in a cubic domain for reasons of code efficiency. The Cartesian computation domain assumes periodic boundary conditions in the $x$ and $y$ directions, so that we are, in effect, simulating an infinite array of vortices, but with the domain widths $L_{x}$ and $L_{y}$ chosen to be sufficiently large that the effects of periodicity are small (see below). However, because the vortex sheds a wake, the ring propagation direction $(z)$ cannot be treated as periodic if interactions with its own wake are to be avoided. We therefore use inflow and outflow conditions, at $z=+L_{z} / 2$ and $-L_{z} / 2$, respectively, and perform the calculations with respect to a moving reference frame attached to the ring. The time-dependent axial velocity $W_{1}(t)$ of this moving reference frame is adjusted by a simple control algorithm to keep the ring at a fixed vertical location within the domain. It thus provides the inflow boundary condition at $z=+L_{z} / 2$, with $w\left(x, y, L_{z} / 2\right)=W_{1}(t)$. (This assumes that $L_{z}$ is large enough, compared to the ring radius $R$, that the velocity induced by the ring vorticity at the inflow plane is negligible.) In order to avoid introducing spurious vorticity into the domain, Neumann conditions are applied to the other two components at $z=+L_{z} / 2$ : $\partial u / \partial z=\partial v / \partial z=0$. At the outflow, $z=-L_{z} / 2$, all three components satisfy a linear gradient condition, with $\partial u / \partial z=\partial v / \partial z=\partial w / \partial z=0$.

Calculation of the reference-frame speed $W_{1}$ (i.e. the time-dependent inflow velocity) requires a time-dependent measure of the vertical ring location $Z$. We use

$$
Z(t)=\frac{1}{2 \Omega} \int z|\omega(t)|^{2} \mathrm{~d} x \mathrm{~d} y \mathrm{~d} z,
$$

where $\Omega$ is the total enstrophy (cf. (2.5)). The value of $W_{1}$ that minimizes the difference between $Z(t)$ and the target location $Z_{c}=0$ is determined by an integral-proportional controller,

$$
W_{1}(t)=2 c_{1}\left(Z(t)-Z_{c}\right)+c_{2}^{2} \int_{0}^{t}\left(Z\left(t^{\prime}\right)-Z_{c}\right) \mathrm{d} t^{\prime}
$$


where $c_{1}$ and $c_{2}$, respectively, set the damping and oscillation time scales. These were chosen to give a critically damped response with $c_{1}=2 \Gamma_{0} / R_{0}^{2}$ and $c_{2}=4 \Gamma_{0} / R_{0}^{2}$ (where the 0 subscript indicates initial values), which locates the ring to within $10^{-3} R_{0}$ of $Z_{c}$ by three $R_{0}^{2} / \Gamma_{0}$ time units, and to within $5 \times 10^{-5} R_{0}$ by $20 R_{0}^{2} / \Gamma_{0}$. Once the ring is locked in place, it remains in the centre of the domain throughout the computation, despite shedding circulation and impulse, and changing its translational velocity.

Since the DNS effectively approximates the motion of a single vortex ring by considering a periodic array of rings in $x$ and $y$, it is necessary to quantify the influence of the implied image vortices, which act to reduce the speed at which the ring propagates, compared to the infinite-domain idealization. The amount of this reduction can be calculated by integrating the net effect of the velocity induced by the neighbouring rings, located at distances $\boldsymbol{x}=\left(i \lambda_{x}, j \lambda_{y}, 0\right)$ from the point in question, where $\lambda_{x}$ and $\lambda_{y}$ are, respectively, the $x$ and $y$ periods of the image array, and $i$ and $j$ are integers. The velocity induced by a single image is $\boldsymbol{U}=\boldsymbol{\nabla} \wedge \boldsymbol{A}$, where $\boldsymbol{A}$ is the far-field velocity potential, which for $|\boldsymbol{x}| \gg R$ is

$$
\boldsymbol{A}=\nabla \wedge\left(\frac{\left(0,0, R^{2} \Gamma\right)}{4|\boldsymbol{x}|}\right) .
$$

The net induced axial velocity $\widetilde{w}$ is thus

$$
\widetilde{w}=-\frac{\Gamma R^{2}}{4 \lambda^{3}} \sum_{i=1, j=1}^{\infty} \frac{i^{2}+j^{2}}{\left(i^{2}+j^{2}\right)^{5 / 2}} \approx-9.032 \frac{\Gamma R^{2}}{4 \lambda^{3}},
$$

where we assume a square array, with $\lambda=\lambda_{x}=\lambda_{y}$, and the $i, j$ summation has been evaluated numerically. This gives both a criterion for choosing the lateral sizes $L_{x}$ and $L_{y}$ of the domain, and also allows the translational velocity exhibited by the ring in the finite-domain simulation to be corrected and compared to the corresponding infinite-domain experimental results. Note that since $\Gamma$ and $R$ vary with time so does the $\widetilde{w}$ correction, and that the ratio of $\widetilde{w}$ to the velocity induced by the local ring within a square domain of size $L$ is $O(R / L)^{3}$.

\subsection{Initial conditions}

The vortex ring is initiated with a Gaussian distribution of vorticity, which is positioned around a centreline path that is perturbed slightly from being perfectly circular, so that written in terms of the distance from the local position of the centreline $R^{\prime}(\theta)$ we have

$$
\omega_{\theta}=\frac{\Gamma}{\pi \delta^{2}} \exp \left(-s^{2} / \delta^{2}\right),
$$

where $s^{2}=z^{2}+\left(r-R^{\prime}(\theta)\right)^{2}$. We suppose that the local radius $R(\theta)$ can be written as a small parameter $\varsigma \ll 1$ multiplied by the sum of a set of $N$ Fourier modes, each with unit amplitude and random phase, so that

$$
\begin{aligned}
R^{\prime}(\theta) & =R_{0}[1+\varsigma f(\theta)], \\
f(\theta) & =\sum_{n=1}^{N} A_{n} \sin (n \theta)+B_{n} \cos (n \theta),
\end{aligned}
$$

where $A_{n}^{2}+B_{n}^{2}=1$. Although this method leads naturally to a divergence-free velocity field, the continuity of the vortex lines themselves is not guaranteed - partly because the vorticity should be tilted slightly to follow the tangent to the path of 


$\begin{array}{ccrccccc}\text { Case } & \delta_{0} / R_{0} & \Gamma_{0} / v & N_{x} \times N_{y} \times N_{z} & t^{*} \Gamma_{0} / R_{0}^{2} & \Gamma^{*} / \Gamma_{0} & R_{\theta}^{*} / R_{0} & \delta_{\theta}^{*} / R_{0} \\ \text { A1 } & 0.413 & 5500 & 256 \times 256 \times 256 & 40.0 & 0.907 & 1.038 & 0.373 \\ \text { A2 } & 0.413 & 10000 & 256 \times 256 \times 256 & 40.0 & 0.911 & 1.038 & 0.360 \\ \text { B1 } & 0.200 & 5500 & 256 \times 256 \times 256 & 25.0 & 0.992 & 0.999 & 0.243 \\ \text { B2 } & 0.200 & 10000 & 384 \times 384 \times 384 & 25.0 & 0.995 & 1.000 & 0.227 \\ \text { B3 } & 0.200 & 7500 & 512 \times 512 \times 512 & 25.0 & 0.993 & 1.000 & 0.232 \\ \text { C1 } & 0.140 & 3000 & 256 \times 256 \times 256 & 15.0 & 0.999 & 0.995 & 0.203\end{array}$

TABLE 1 . Run parameters. The * superscript indicates quantities at the sampling initiation time $t^{*}$.

the vortex centreline, and partly because the implied cross-section area of the vortex is not perfectly constant around the ring. The above vorticity field can be corrected to become divergence-free by superimposing the gradient of a scalar field $\nabla \phi$ and requiring that $\phi$ satisfy a Poisson equation whose source term is the divergence error of the original vorticity field. The complete initial field is then obtained by solving for the vector streamfunction that is consistent with the vorticity distribution, and the velocity field then follows directly by taking its curl. The method of perturbing the geometry of the ring, rather than the velocity field, produces a modal energy spectrum that decays with the second power of $n$.

\section{Results}

\subsection{Cases and quality diagnostics}

The parameters that define the six cases summarized in table 1 allow consideration of the effects of both Reynolds number $R e=\Gamma_{0} / \nu$ and the slenderness ratio $\epsilon_{0}=\delta_{0} / R_{0}$. Since this study will revisit some of the issues first raised by Shariff et al. (1994), two of our runs have been designed to match theirs. The Case A1 and B1 values, respectively, correspond to those used for their Runs 3 and 12 , with $R e=5500$ for both and $\epsilon_{0} \approx 0.4$ and $\approx 0.2$, defining rings within the thick- and thin-core regimes. Cases A2 and B2 are higher-Reynolds-number versions of A1 and B1, respectively, with $R e=10000$. Case B3 defines another $\epsilon_{0}=0.2$ thin-core ring, at an intermediate Reynolds number, $R e=7500$. This case, with its intermediate $R e$ and higher spatial resolution, will be especially useful when we examine the nonlinear breakdown and the early stages of the fully turbulent regime. Finally, Case C1 was designed to help determine the expression for the translational velocity constant $C$ (see $\S 3.2$ ), by capturing both thin- and thick-core behaviour. The number of grid cells employed in each direction $\left(N_{x}, N_{y}, N_{z}\right)$ is shown in table 1 . The quality of the spatial resolution is examined below. A cubic Cartesian domain was used for all cases, with $L_{x} / R_{0}=L_{y} / R_{0}=L_{z} / R_{0}=8$. Equation (2.9) implies that this introduces differences between the finite- and infinite-domain ring translational velocity of approximately $0.005 \Gamma_{0} / R_{0}$. All cases also used a constant time step, with the Courant-FriedrichsLewy (CFL) number, based on local velocity and grid size, always less than 0.15 . A radius perturbation of amplitude $\zeta=2 \times 10^{-4}$ was imposed on the first 32 azimuthal modes for the thin-core rings (Cases B1, B2, B3 and C1), while for (thick-core) Cases A1 and A2 the first 24 modes were disturbed, following the approach of Shariff et al. (1994).

The reliability and accuracy of the present code was validated by comparing the results of Case A1 with laminar and linear-instability results obtained with an 
(a)

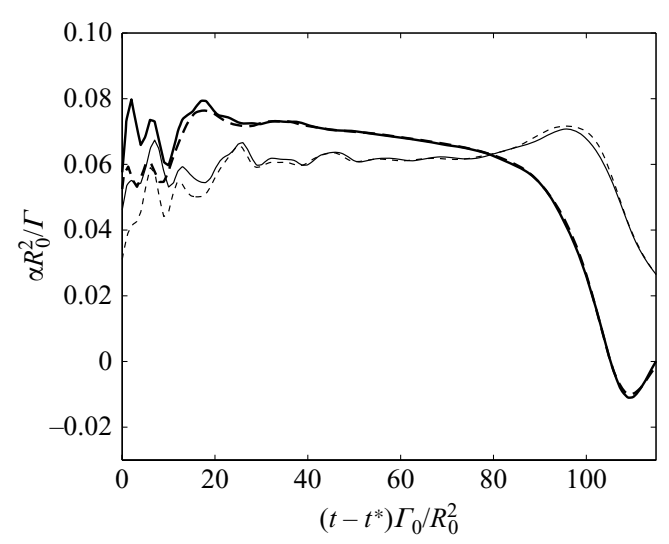

(b)

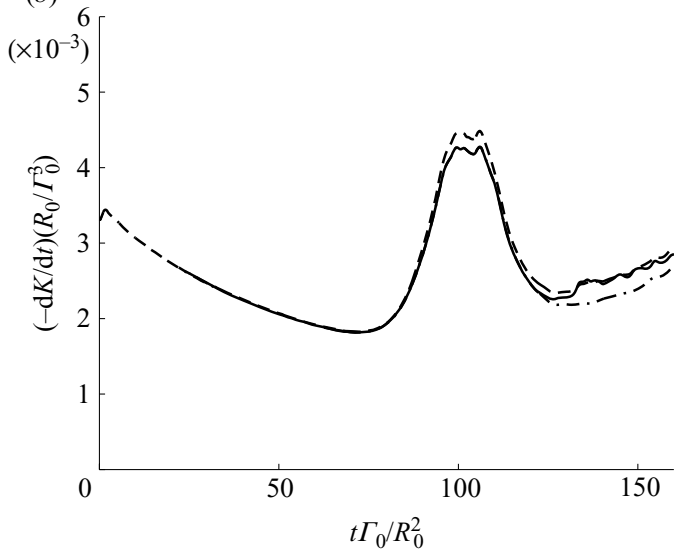

Figure 2. Numerical integrity. (a) Comparison of modal growth rates for azimuthal modes $n=5$ (thin line) and $n=6$ (thick line): - Case A1;---, $192 \times 192 \times 768$ spectral DNS. (b) History of rate of change of volume-integrated kinetic energy $K$ for Case B3 (thin core): ,$----\mathrm{d} K / \mathrm{d} t ;---, \epsilon_{K} ;-, \epsilon_{K}-F_{K}$. Dissipation and flux terms shown for $t \geqslant t^{*}$, where $t^{*}$ is the sampling initiation time (see $\S 3.2$ ).

in-house fully spectral third-order Runge-Kutta code $\uparrow$ using $192 \times 192 \times 768$ Fourier modesf. The spectral domain was large enough $\left(L_{z}=32 R_{0}\right)$ in the $z$-direction to prevent the ring interacting with its wake during the time considered. (Were it not for the need to employ non-periodic boundary conditions in $z$, the fully spectral code could have been used for this study.) Excellent agreement was found for the ring translational velocity and integral measures, as well as the linear-regime growth rates of the azimuthal disturbances, which after adjusting to a slightly different random initialization eventually track each other (see figure $2 a$ ). A domain sensitivity study was also undertaken whereby Case A1 was repeated on two larger domains, one expanded laterally in both $x$ and $y$ by $50 \%$, and the other increased in the $z$ direction by $100 \%$. The ring integral measures were found to differ by less than $0.5 \%$, and the azimuthal growth rates by less than $1 \%$.

A further check of the spatial resolution is provided in figure $2(b)$, which shows histories of the rate of change of the volume-integrated kinetic energy $K$ (per unit mass) within the simulation domain, with respect to the frame of reference attached to the ring. $\Phi$ Within an infinite domain, the rate of change of $K$ is equal to the volumeintegrated rate of kinetic energy dissipation $-\epsilon_{K}$. However, since kinetic energy is lost to the wake and convected out of the finite domain, here the energy balance is altered such that

$$
\frac{\mathrm{d} K}{\mathrm{~d} t}=-\epsilon_{K}+F_{K}
$$

where $F_{K}$ is the net volume-integrated kinetic energy flux, set by the difference between the (unsteady) fluxes at the inlet and outlet planes. The difference between the left- and right-hand sides of (3.1) is a measure of the spatial discretization error in resolving the

$\dagger$ Written and run by Dr C. P. Yorke of University of Southampton.

$\ddagger$ This involved a $288^{3}$ collocation grid, to allow de-aliasing of the spatial derivatives.

I The moving coordinate system and the associated unsteady inflow define an effective pressure gradient of $-\mathrm{d} W_{1} / \mathrm{d} t$, such that $K=\frac{1}{2} \int_{V}\left(u_{i} u_{i}-W_{1}^{2}\right) \mathrm{d} V$, where $V$ is the volume of the DNS domain. 


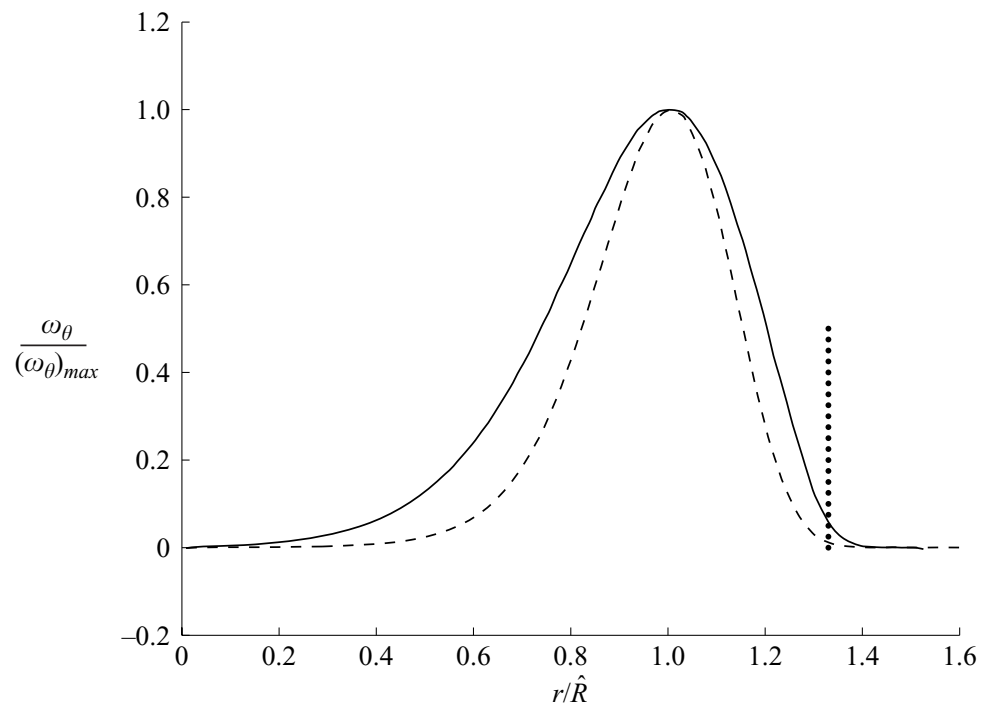

FiguRE 3. Comparison of core vorticity distributions for a thick- and a thin-core ring, at $t=t^{*}:-$, Case A1 $\left(\epsilon_{0}=0.41\right) ;---$, Case B1 $\left(\epsilon_{0}=0.20\right)$. The broken vertical line indicates the location of the instantaneous zero streamline on the concurrent plane. Radial locations non-dimensionalized by local $\widehat{R}$, the distance from the origin to the peak in $\omega_{\theta}$.

smallest turbulence scales. For all six cases, the difference was within $1 \times 10^{-5} \Gamma_{0}^{3} / R_{0}$ up to the point of transition. Transition involves stretching of the vorticity filaments to fine scale, resulting in an enstrophy and dissipation peak. Note that the flux term $F_{K}$ is only significant after the ring breaks down and its turbulent wake reaches the outflow boundary (compare the solid and chain dot curves in figure $2 b$ ). Even during this most difficult to resolve phase $\left(t>80 R_{0}^{2} / \Gamma_{0}\right)$, the accuracy is reasonable, with the error remaining less than $2.2 \times 10^{-4} \Gamma_{0}^{3} / R_{0}$.

\subsection{Laminar evolution}

The initial Gaussian distribution of vorticity is only an exact steady solution in the limit of infinitely thin cores. Since the Gaussian cores specified here have finite size (and thus only approximately satisfy the Navier-Stokes equations) the ring initially undergoes an 'equilibration' phase, during which the $\omega_{\theta}$ profile across the core region relaxes towards a new equilibrium state, as noted by Shariff et al. (1994). This is obtained after the Gaussian profile approaches the axisymmetric inviscid ideal for which, in axes attached to the ring, $\omega_{\theta} / r$ is solely a function of the streamfunction $\psi$. The core profile must thus depart from the initially symmetric Gaussian distribution, and become skewed, with $\omega_{\theta}$ decreasing faster toward the bubble edge than the ring centre. The difference between the inner and outer vorticity distributions increases as the slenderness ratio $\epsilon$ increases (figure 3). During the equilibration phase $\omega_{\theta}$ is shed from the ring, reducing the circulation and modifying the ring geometry; this process is most severe for thick-core rings. These observations led us to define a sampling initiation time $t^{*}$ at a time just after equilibration has occurred, such that the core vorticity distribution has fully adjusted and the associated shed vorticity has left the logging domain, thereby avoiding spurious measurements of the ring characteristics. (The impact of the start-up wake as it passes out of the logging domain can be seen in the local increase in circulation decay rate between $t=5$ and $15 R_{0}^{2} / \Gamma_{0}$ in figure $4 a$ ). 
(a)

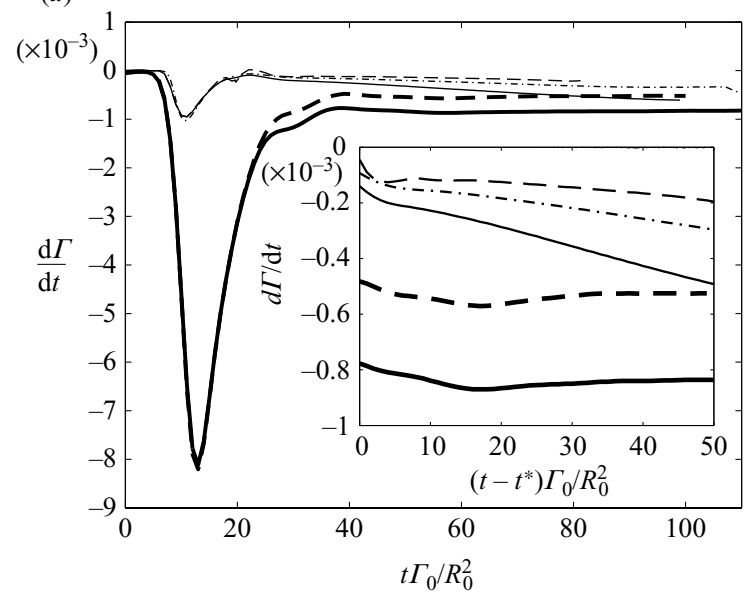

(b)

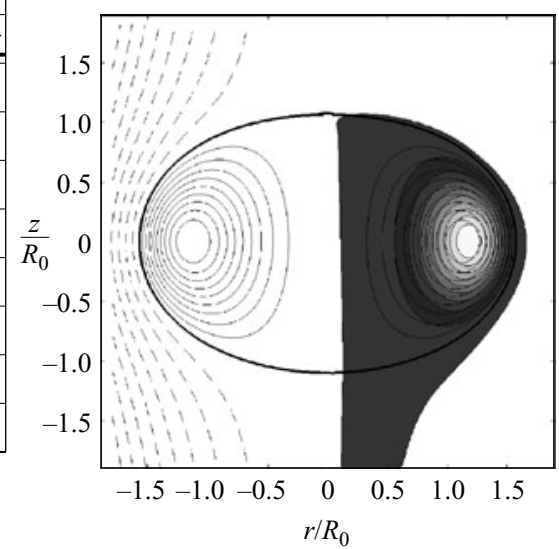

Figure 4. (a) Histories of rate of change of circulation $\mathrm{d} \Gamma / \mathrm{d} t:-$, Case A1 (thicker line) and B1 (thinner); ---, Case A2 (thicker) and B2 (thinner); - - - Case B3. (b) Loss of of azimuthal vorticity $\omega_{\theta}$ for Case A1 at $\left(t-t_{*}\right) \Gamma_{0} / R_{0}^{2}=20:-, \psi \leqslant 0$ (thicker contour denotes $\psi=0$ ); ---, $\psi>0$; shaded contours show $\omega_{\theta}$ (darker contours show weaker vorticity).

The initial sampling time is documented together with the ring parameters associated with this time (indicated by an asterisk) in table 1.

Having adjusted, the $\omega_{\theta}$ profile extends outside the instantaneous zero streamline (broken vertical line, see figure 3) for both the thin- and thick-core rings, but to a far greater degree for the thick-core ring. Vorticity extending across the zero streamline is nominally outside the entrainment bubble. It can then either be entrained back into the bubble or cross the bubble surface and depart into the wake, as suggested by Maxworthy's model for diffusive entrainment (Maxworthy 1972). Figure 4(b) shows precisely this mechanism, with weak vorticity (shaded contours on the right-hand side) trailing into the wake over the zero streamline (thick-solid contour). The reason why vorticity crossing the instantaneous $\psi=0$ streamline can be entrained back into the entrainment bubble is that the zero streamline is not stationary and moves in time to incorporate changes to the ring translational velocity and integral parameters. Lagrangian analysis of the flow yields an entrainment bubble surface which takes into account the moving $\psi=0$ streamline. Vorticity diffused across this boundary, outboard of the zero streamline, passes into the wake. These arguments are explored further in $\S 3.5$. Whether the 'leaked' vorticity is recovered or not, the vorticity profile is clipped by the presence of the entrainment bubble surface. This vorticity clipping leads to a sharpening in the $\omega_{\theta}$ profile in the vicinity of the zero streamline surface, hence increasing the skewness of the core. For both cases, the instantaneous zero streamline surface appears at a constant distance $r \approx 1.33 \widehat{R}$ from the ring centreline, where $\widehat{R}$ is the current radial location of the $\omega_{\theta}$ peak. The instantaneous zero streamline surface thus expands to track radial displacement of the vorticity peak as the core diffuses, leading to a relationship between the core thickness and the rate of decay of $\omega_{\theta}$.

The loss of $\omega_{\theta}$ manifests itself in an overall reduction of ring circulation. Figure 4(a) displays the rate of decay in circulation during the laminar regime. The main figure shows the rate of decay from the start of the simulation, with the early peaks corresponding to the vorticity shed during the core adjustment leaving the domain. 
(a)

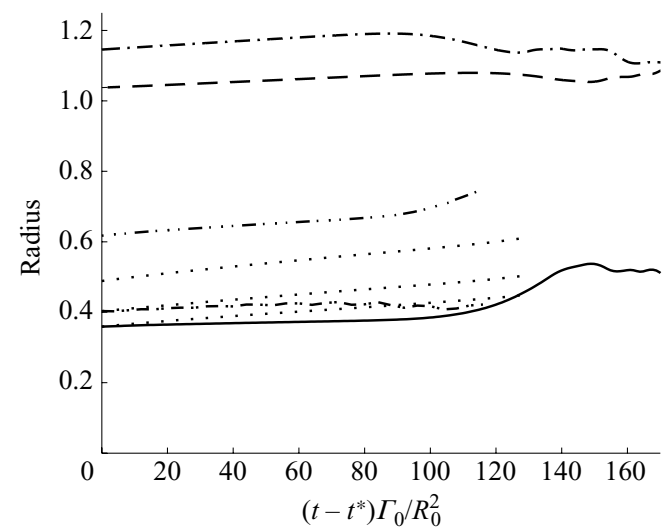

(b)

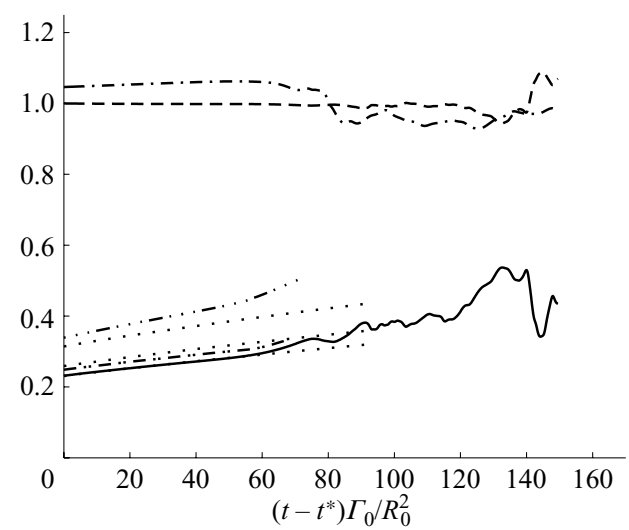

Figure 5. Histories of core and ring radii for $(a)$ Case A1 (thick core) and $(b)$ Case B3 (thin core):,$- \delta_{\theta} / R_{\theta} ;-\cdots-, a_{1} / R_{\theta} ;-\cdots-, a_{e} / R_{\theta} ; \cdots$, theoretical core diffusion determined by (2.6) non-dimensionalized by $R_{\theta}$, (with the lower line corresponding to $\delta_{\theta}$, the middle to $a_{1}$ and the upper to $\left.a_{e}\right) ;---, R_{\theta} / R_{0} ;-\cdot-, R_{\Omega} / R_{0}$.

We focus on the inner figure which presents the behaviour when the core has adjusted its vorticity distribution, after $t^{*}$. Comparing Cases A1 with B1 and A2 with B2, we see that despite similar initial Reynolds numbers $\Gamma^{*} / v$, the thick-core rings reduce in circulation faster than the thin-core rings. This can be attributed to the increased skewness of the thick-core rings, resulting in greater $\omega_{\theta}$ extension across the entrainment bubble surface. The rate of circulation loss increases with time for the thin-core rings, which is consistent with the ongoing core diffusion, such that they become increasingly skewed. The thick-core rings, on the other hand, exhibit a larger but more nearly constant rate of circulation loss, since they are closer to a quasi-steady state defined by fixed $\epsilon$ (cf. figures $5 a$ and $5 b$ ), and thus experience little change to their skewness and associated relative rate of $\omega_{\theta}$ diffusion.

Maxworthy's model for diffusive entrainment (Maxworthy 1972) predicts a $-2 / 3$ power law decay of $\Gamma$. The decay rate was not found to be universal by Dabiri \& Gharib (2004) however, who experimentally generated rings at Reynolds numbers between 2000 and 4000, finding that $\Gamma$ decayed with power laws between -0.27 and -0.067 , with the power decreasing with increasing Reynolds number. We find for the present rings that the circulation tends to decay as $\Gamma \sim t^{c}$ where $c$ is in the range -0.01 to -0.002 . We can account for the range of reported circulation decay rates by the difference in Reynolds numbers across the previous and present cases. As shown in figure 4(a), for identical initial $\epsilon$, the decay rate increases monotonically with decreasing Reynold number.

The laminar evolution of the core radius measures, $\delta_{\theta}, a_{e}$ and $a_{1}$, normalized by the instantaneous value of $R_{\theta}$, and the ring radii measures $R_{\theta}$ and $R_{\Omega}$ are shown in figure 5, for two rings that typify the behaviour of thin and thick cores (Cases A1 and B3). To calculate $a_{1}$, the core centre was first determined by linear interpolation, then second-order polynomial extrapolation was used to find the distance from the core centre to the location of the maximum tangential velocity on the plane $z=0$ inboard and outboard of the core centre. These two lengths were then averaged to give $a_{1}$. 
Since the integral measure $R_{\Omega}$ is weighted by $|\omega|^{2}$, it is biased toward regions of high vorticity and hence approximates the radial location of peak vorticity. On the other hand, $R_{\theta}$ (being weighted by $\omega_{\theta}$ ) effectively yields the radial location of the centre of vorticity of the core, which during the laminar regime is always inboard of $R_{\Omega}$, owing to the skewness of the core $\omega_{\theta}$ profile. The difference between the two measures is thus an indication of the core skewness, which is greater for thicker cores. Note that $R_{\theta}$ for the thin-core rings is approximately constant, while $R_{\Omega}$ increases slightly with time, demonstrating the increasing skewness of the thin-core rings owing to core diffusion. The thick-core rings behave differently, in that both $R_{\theta}$ and $R_{\Omega}$ increase with time. This shows that the ring is expanding radially while the skewness of the core remains approximately constant.

The three dotted lines in figures $5(a)$ and $5(b)$ represent the predicted core diffusion for an equivalent core with a Gaussian profile in accordance with (2.6). The history of the thin-core ring follows the Saffman diffusion equation very closely, with the exception of $a_{e} / R_{\theta}$, which deviates with time away from the upper dotted line. The divergence can be attributed to the increasing core skewness. As the core $\omega_{\theta}$ distribution becomes less Gaussian, the translational velocity of the ring reduces with an associated increase in $a_{e}$ (cf. (2.1)). For the thick-core ring, the slenderness ratios $a_{1} / R_{\theta}$ and $\delta_{\theta} / R_{\theta}$ remain approximately constant with time. The core expansion is constrained by the ring geometry, as $\omega_{\theta}$ must equal zero on the ring centreline and its outer extent is clipped by the zero entrainment streamline (which expands at the same rate as the radius). Consequentially the core diffuses at a similar rate to the ring radius, leading to a nearly constant $\epsilon$. With both $\epsilon$ and hence the degree of skewness nearly constant, the core distribution of vorticity changes little, resulting in the nearly constant rate of decay of circulation for thick cores seen in figure $4(a)$.

The transitional and early turbulent behaviour of the ring is also presented in figure 5, to give perspective to the laminar results. Transition begins near $\left(t-t^{*}\right) \Gamma_{0} /$ $R_{0}^{2}=95$ and 55 for Cases A1 and B3, respectively, with the decrease of $R_{\Omega}$ as the core distorts and the development of interior and peripheral vortical structures moves the location of the maximum vorticity. The transitional and early turbulent behaviour will be examined further in $\S 3.4$.

Histories of the translational velocity $U$ of the thick- and thin-core rings are shown in figures $6(a)$ and $6(b)$, respectively, and compared to equivalent rings with a Gaussian core profile. These include the periodic correction $\widetilde{w}$ (cf. (2.9)) to account for the induced velocity of the periodic array of vortices, so we in effect report the translational velocity of a single vortex ring in an unbounded domain. The Gaussian ring velocity $U_{\mathrm{G}}$ is given by (2.1), where $C=-0.558$ and the instantaneous values of $\Gamma, R_{\theta}$ and $\delta_{\theta}$ are employed. The difference between $U_{\mathrm{G}}$ and the numerical results reveal the impact of the non-Gaussian core $\omega_{\theta}$ distribution. The difference is greater for the highly skewed thick-core ring and remains almost constant as the core diffusion is limited (for reasons given above). Since for the thin-core ring the $\omega_{\theta}$ distribution becomes increasingly skewed as the simulation progresses, the difference between the Gaussian and the DNS velocity increases with time. For all cases the rings decelerated smoothly until the onset of turbulence, whereby the translational velocity fluctuates dramatically as it decays.

The inclusion of $C$ in (2.1) allows for cores with finite slenderness ratios $\epsilon$ that possess non-Gaussian core distributions. The increasing difference between the ring translational velocity and that of a Gaussian-core ring implies that $C$ becomes increasingly negative with increasing $\epsilon$. We might expect that the effect of increasing 
(a)

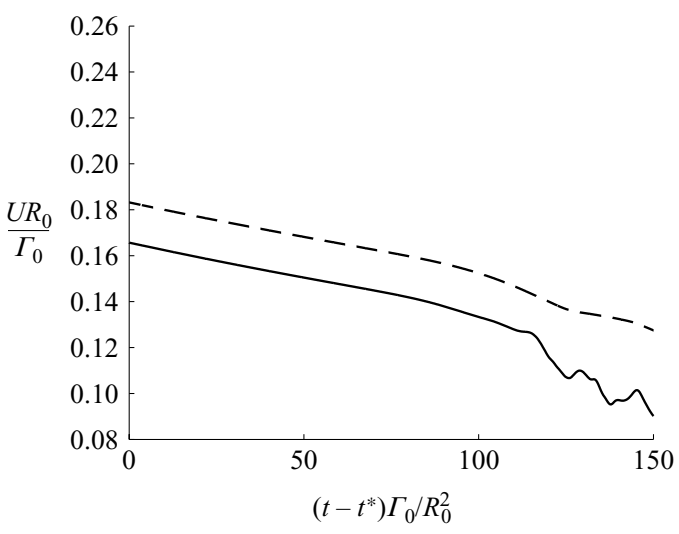

(b)

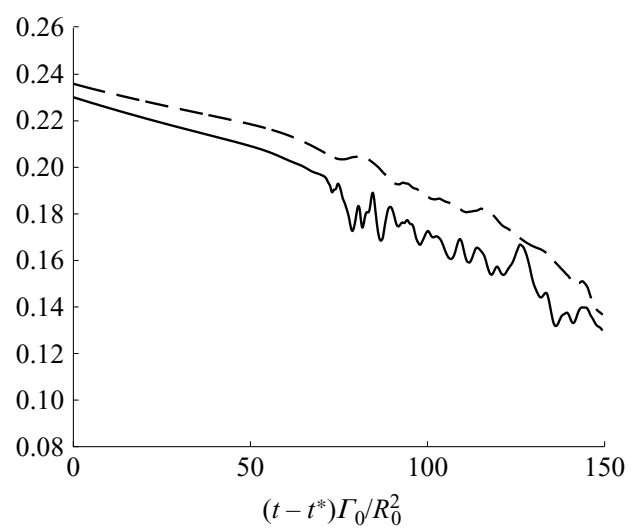

Figure 6. Histories of vortex translational velocity for (a) Case A1 (thick core) and (b) Case B3 (thin core) : ---, $U_{\mathrm{G}} ;-, U$.

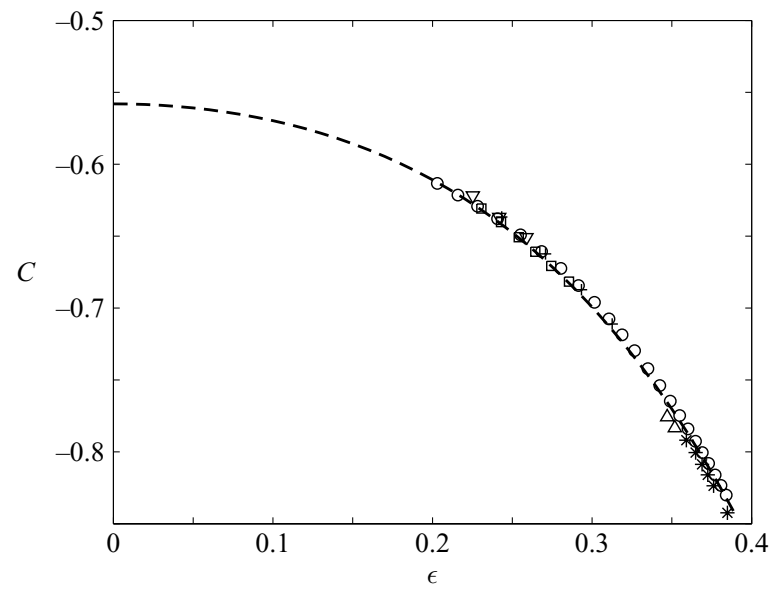

Figure 7. Translational velocity parameter $C$ vs. $\epsilon$ :---, $C=-1.12 \epsilon^{2}-5.0 \epsilon^{4}-0.558$;

*, Case A1; $\triangle$, Case A2; +, Case B1; $\nabla$, Case B2; $\square$, Case B3; O, Case C1.

skewness would manifest itself as an even-series expansion in $\epsilon^{2}$, i.e. $C$ can be approximated as $C=A \epsilon^{2}+B \epsilon^{4}+C^{\prime}$, where the constant $C^{\prime}$ must equal -0.558 , since for small finite $\epsilon, C$ must equal -0.558 . The parameter $C$ has been extracted from the simulations and is plotted in figure 7 against the instantaneous value of $\epsilon=\delta_{\theta} / R_{\theta}$ for all cases. The results are well approximated by $C=-1.12 \epsilon^{2}-5.0 \epsilon^{4}-0.558$ (dashed curve). The generalized expression for the translation speed of a laminar vortex ring as a function of $\Gamma, R$ and $\epsilon$ then becomes

$$
U=\frac{\Gamma}{4 \pi R}\left[\ln \left(\frac{8}{\epsilon}\right)+A \epsilon^{2}+B \epsilon^{4}+C^{\prime}+\cdots\right],
$$

where $A=-1.12, B=-5.0$ and $C^{\prime}=-0.558$. 


\subsection{Linear growth of azimuthal instability}

During the initial laminar phase, an azimuthal instability associated with the vortex core develops, and deforms the core into a standing wave with an integer number $n$ of waves around the circumference. The number of modes depends on the slenderness ratio such that $n \sim 2.26 / \epsilon$ (for a Gaussian core distribution of vorticity, Shariff et al. 1994). The inviscid case was examined by Widnall \& Tsai (1977) and the growth rate $\alpha_{W T}$ given by

$$
\alpha_{W T}=\frac{\Gamma}{4 \pi R^{2}}\left[\left(0.856 \ln \left(\frac{8 R}{a_{1}}\right)-0.9102\right)^{2}-0.4535\right]^{1 / 2} .
$$

(Note the last term differs from its original form in Widnall \& Tsai (1977) to reflect the error corrected in Shariff et al. 1994.) The growth rate for a mode $n$ is defined as $\alpha_{n} \equiv \frac{1}{2}\left(1 / E_{n}\right) \mathrm{d} E_{n} / \mathrm{d} t$, where $E_{n}$ is the energy of the mode. However, the numerical work of Shariff et al. (1994) found that the effect of viscosity is to reduce the growth rate from the inviscid value by a factor that depends on the local internal Reynolds number of the core, and established the viscous correction factor with

$$
\alpha_{S}(\beta)=\alpha_{W T}(\beta)\left[1-\frac{\widehat{\alpha}_{1}(\beta)}{R e_{S}}\right],
$$

where $\alpha_{S}$ is the adjusted growth rate, $\widehat{\alpha}$ is a non-dimensional coefficient which in principle depends on $\beta=a_{1} / R$, and the internal Reynolds number $R e_{S}=e a_{1}^{2} / v$ is the ratio of the local induced strain field $e$ to viscous damping, where Saffman's estimate of the strain rate (Saffman 1978)

$$
e=\frac{3 \Gamma}{16 \pi R^{2}}\left[\ln \left(\frac{8 R}{a_{e}}\right)-\frac{17}{12}\right]
$$

is used. Shariff et al. formulated the viscous correction by comparing the measured growth rates for a number of rings with $\epsilon$ varying from 0.2066 to 0.4131 , and across a range of Reynolds numbers $R e$ from 1200 to 10000 . They determined the growth rate as being that of the most amplified azimuthal mode (i.e. the mode with the largest growth rate at a given time, not necessarily the mode currently with the most energy) observed at a time $\bar{t} \Gamma_{0} / R_{0}^{2}$ between 52.5 and 57.5. The growth rate was averaged over $15 R_{0}^{2} / \Gamma_{0}$ time units centred about $\bar{t}$. However, as the vortex ring geometry changes slowly over time because of viscous diffusion of the core, the most amplified mode will also slowly change. In fact, successive modes with reducing $n$ will be selected and amplified, so that the average growth rate measured by this method can be expected to be slightly lower than suggested by the equation above.

Shariff et al. estimated the internal Reynolds number $R_{S}$ at time $\bar{t}$ using the core measures $a_{1}$ and $a_{e}$, and assuming a Gaussian core distribution of vorticity diffusing in accordance with (2.6) (accurate for thin cores but less so for thicker ones). Finally, $\alpha_{W T}$ was calculated using the initial ring parameters and it was found that $\widehat{\alpha}_{1}=18$ fitted their results well.

The main differences between our numerical investigation and the simulations of Shariff et al. are that our boundary conditions are not periodic in the direction of ring propagation and that we use a cubic domain rather than a cylindrical one. The latter requires us to interpolate our velocity fields onto a cylindrical grid in order to extract the modal energy spectrum and associated modal growth rates. An azimuthal Fourier transform was performed over the cylindrical grid to reveal the modal energy histories displayed in figure 8 . We follow Shariff et al. and report the growth rate of the most 


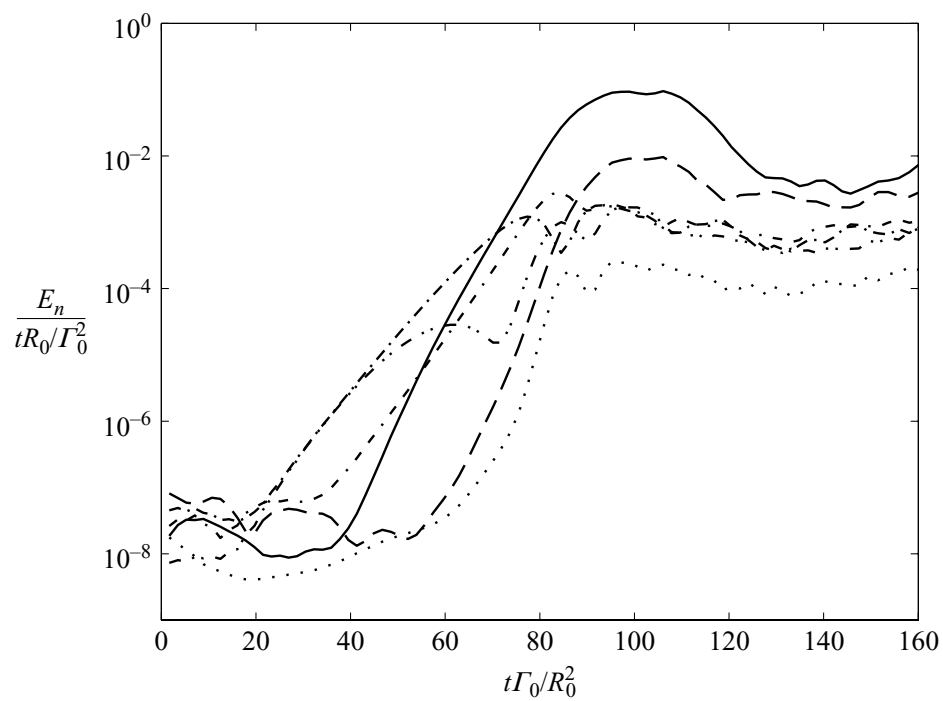

FIGURE 8. Evolution of selected azimuthal modal energies for Case B3:-,$- n=1$; ,$--- 2 ;-\cdot-, 9 ;-\cdot-, 10 ;-\cdots-, 11 ; \cdots, 18$.

$\begin{array}{ccccccccccc}\text { Case } & \bar{t} & n(\bar{t}) & \alpha & \alpha_{S} & \Gamma(\bar{t}) & R_{\Omega}(\bar{t}) & a_{e}(\bar{t}) & a_{1}(\bar{t}) & \alpha_{W T}(\bar{t}) & \alpha_{S}(\bar{t}) \\ \text { A1 } & 52.5 & 6 & 0.072 & 0.090 & 0.897 & 1.173 & 0.65 & 0.44 & 0.081 & 0.069 \\ \text { A2 } & 52.5 & 6 & 0.082 & 0.098 & 0.904 & 1.144 & 0.62 & 0.42 & 0.087 & 0.080 \\ \text { B1 } & 52.5 & 9 & 0.099 & 0.108 & 0.989 & 1.062 & 0.42 & 0.30 & 0.128 & 0.103 \\ \text { B2 } & 47.5 & 10 & 0.130 & 0.126 & 0.995 & 1.044 & 0.34 & 0.26 & 0.141 & 0.124 \\ \text { B3 } & 52.5 & 9 & 0.112 & 0.119 & 0.989 & 1.056 & 0.39 & 0.28 & 0.133 & 0.113\end{array}$

TABLE 2. Comparison of present growth rate $\alpha$ with the viscous prediction $\alpha_{S}$ of Shariff et al. (1994). Time $\bar{t}$ given in units of $R_{0}^{2} / \Gamma_{0}$, growth rates in units of $\Gamma_{0} / R_{0}^{2}$, circulation in units of $\Gamma_{0}$ and lengths in units of $R_{0}$.

amplified mode at $\bar{t} \Gamma_{0} / R_{0}^{2}=52.5$, averaging the growth rate over a time window of $\pm 7.5 R_{0}^{2} / \Gamma_{0}$ centred at $\bar{t}$. The Case B2 result, however, is reported at the earlier time of $\bar{t} \Gamma_{0} / R_{0}^{2}=47.5$ to avoid the possibility of being affected by the nonlinear regime. The growth rate $\alpha_{S}$ was calculated using the instantaneous values of $a_{1}$ and $a_{e}$ at $\bar{t}$ as approximated by (2.6) and $\Gamma_{0}$ and $R_{0}$ in accordance with the methodology used by Shariff et al.

A comparison between the present growth rates $\alpha$ and those given by the Shariff et al. (1994) viscous correction $\alpha_{S}$ is presented in table 2. It is found that $\alpha_{S}$ is within $9 \%$ of $\alpha$ for the thin-core rings, Cases B1, B2 and B3. However, the difference is greater for the thick-core cases, A1 and A2, at approximately $25 \%$. The difference in growth rates for the thick-core rings can be attributed in part to the periodicity of the Shariff et al. simulations in the direction of ring propagation. Since we follow essentially identical initialization procedures, the Shariff et al. rings were subject to the same equilibrilisation phase as documented here. In Shariff et al.'s triply periodic computations; the shed vorticity associated with the vortex ring adjusting to its steadystate vorticity profile ( see $\S 3.2$ ) is ejected from the ring during the equilibration phase and cycles periodically through the domain, encountering the ring on each pass 


$\begin{array}{cccccc}\text { Ring } & \alpha_{D} & \alpha_{W T, D} & \alpha_{S, D} & \alpha_{W T}^{\prime} & \alpha_{S}^{\prime} \\ \mathrm{A}^{\prime} & 0.78 & 1.36 & 1.00 & 1.39 & 1.02 \\ \mathrm{C}^{\prime} & 0.91 & 1.59 & 1.03 & 1.39 & 0.90\end{array}$

TABLE 3. Experimental growth rates $\alpha_{D}$ of Dazin et al. (2006a) and recorded quantities $\alpha_{W T, D}$ and $\alpha_{S, D}$ compared to $\alpha_{W T}^{\prime}$ and $\alpha_{S}^{\prime}$ which result from using $a_{1}$ in (3.3). Growth rates given in $\mathrm{s}^{-1}$.

and providing a forcing of the ring instability. This explanation was verified using the in-house spectral DNS code mentioned earlier, periodic in all three directions, by initializing a thick-core ring in domains of different length. The longest domain ensured breakdown occurred before the wake interacted with the vortex ring and yielded a growth rate $10 \%$ less than a domain of similar size to that used by Shariff et al. The growth rate for thin cores is more accurate because the initial equilibration wake shedding is negligible, thus the rings are not influenced in the same way. As shown in $\$ 3.2$, all ring measurements vary during the laminar regime, dependent on $\epsilon$ and the Reynolds number. This led us to test the Shariff et al. viscous correction by incorporating the instantaneous $\Gamma(\bar{t}), R_{\Omega}(\bar{t}), a_{1}(\bar{t})$ and $a_{e}(\bar{t})$ at $\bar{t}$ to estimate $\alpha_{W T}(\bar{t})$, $e(\bar{t})$ and $\operatorname{Re}_{S}(\bar{t})$, with $\alpha_{S}(\bar{t})=\alpha_{W T}(\bar{t})\left[1-\widehat{\alpha}_{1} / \operatorname{Re}_{S}(\bar{t})\right]$. Using this method with $\widehat{\alpha}_{1}=8$ improves the agreement to within $5 \%$ for both the thin- and thick-core cases. This supports the validity of correcting the inviscid growth rate based on the internal Reynolds number $\operatorname{Re}_{S}$.

Dazin et al. (2006a) used PIV to calculate the instability growth rate of two of their experimental rings $\mathrm{A}^{\prime}$ and $\mathrm{C}^{\prime}$, reporting differences with their implementation of the Shariff et al. correction. However, they followed a slightly different method to that proposed by Shariff et al. using $a_{e}$ as their core measure in place of $a_{1}$ in calculating $\alpha_{W T}$. We have followed the methodology of Shariff $e t$ al. to give a corrected prediction for their growth rates, $\alpha_{W T}^{\prime}$ and $\alpha_{S}^{\prime}$ (see table 3). The difference between the growth rate of ring $\mathrm{C}^{\prime}$ and $\alpha_{S}$ is reduced significantly; however, for ring $\mathrm{A}^{\prime}$ the difference is still large. Another important difference between the two methods is the sampling period over which the growth rate is calculated. Dazin et al. had access to few sample points over the entire laminar regime with which to calculate the growth rate. Figure 8 shows that this simplification is reasonable for mode 9, which grows at an approximately constant rate, but much worse for modes 10 and 11, as their growth rate changes greatly during the laminar regime. In general, the growth rate of a single mode will not be constant throughout the laminar regime, as viscosity diffuses the core region amplifying modes with monotonically decreasing mode number.

\subsection{Nonlinear transition phase}

The elliptical instability initially leads to a narrow band of modes growing independently, which determine the number of standing waves around the core azimuth. However, at a certain amplitude the modes begin to interact nonlinearly with each other producing higher harmonics and lower-order intermodulation products (see figure 8), consistent with the experiments of Dazin et al. (2006b) and the simulations of Shariff et al. (1994). Constructive interference between neighbouring modes causes a noticeable 'lop-sidedness' to the wave growth and associated core displacement, which is represented by the rapid growth of the $n=1$ mode prior to transition. The relative dominance of the $n=1$ mode varies from case to case and is largest for the thin-core Cases B1, B2 and B3, which during the nonlinear phase have 8,10 and 9 waves 


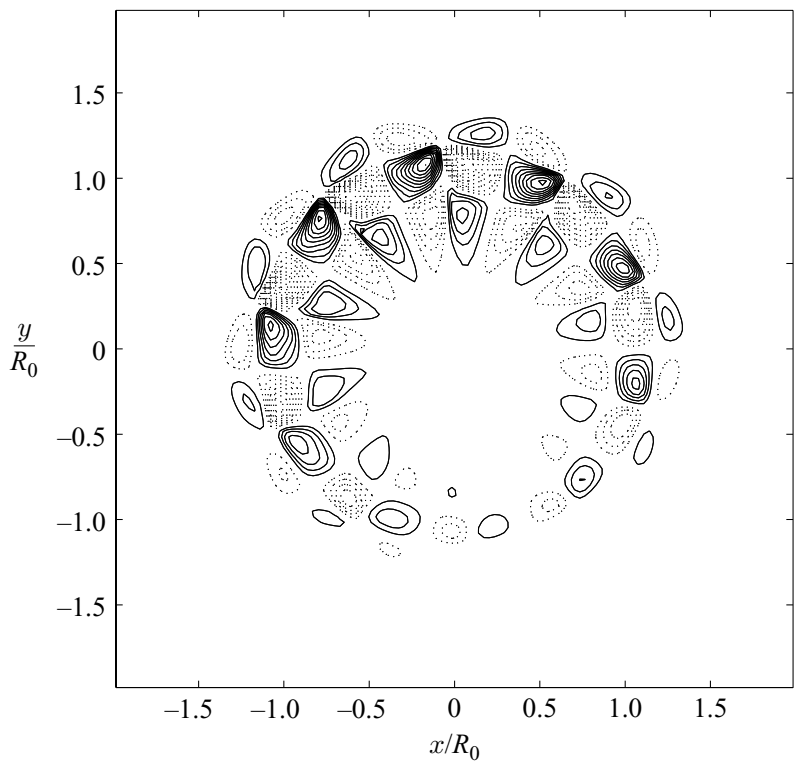

FIGURE 9. Contours of $\omega_{z}$ on the horizontal plane through the centre of the ring $(z=0)$ for Case B3 at time $\left(t-t^{*}\right) \Gamma_{0} / R_{0}^{2}=55:-, \omega_{z}>0 ; \cdots, \omega_{z}<0$. Contour increments at $\left|\omega_{z \max }\right| / 10$.

around the core, respectively. An $n=0$ mode also grows rapidly in the azimuthal velocity energy component (i.e. an axial flow along the circumferential axis of the vortex core), which corresponds to a mean azimuthal profile of opposing streams, as documented by Shariff et al. (1994) and shown in their figure 7. A description of the nature of this axial flow follows in $\S 3.6$.

Here we make a distinction between the region of intense vorticity at the core centre, which we call the 'inner core' and the surrounding outer-core region of lower vorticity, which we call the 'halo' vorticity. The elliptical instability causes displacement of the inner core into a stationary wave pattern, while the halo vorticity displaces in the opposite direction, consistent with the second radial mode (Widnall 1975). In a slice across the $z=0$ plane, the signature of the second radial mode in the axial and radial components of vorticity is three layers arranged radially (figure 9). The halo vorticity occupies the inner and outer layers and is $180^{\circ}$ out of phase with the inner core. The structure is also apparent in three-dimensional isosurface plots of the second invariant of the velocity gradient tensor II (a useful marker of vortical structure), and isosurface plots of vorticity shown in figure 10. They show that as the inner-core displacement becomes appreciable, the halo vorticity rolls up into an interwoven mesh of secondary structure. The secondary structure develops first in the top left-hand corner of figure 10( $a)$ (bottom of figure 10b), where constructive interference between the azimuthal modes $n=9$ and $n=10$ yields the greatest inner-core displacement. It then develops around the azimuth of the ring as the local inner-core displacement increases with time. The figures further show that the secondary structure consists of a series of loops which encompass the inner core. The inner core weaves its way through the centre of the loops. It is noted that neighbouring loops are of alternating signed vorticity, consistent with the observations of Dazin et al. (2006b) and simulations of Bergdorf et al. (2007), and are formed by tilting and reorganization of the halo vorticity. The loops touch at saddle points positioned in azimuthal planes aligned 
(a)

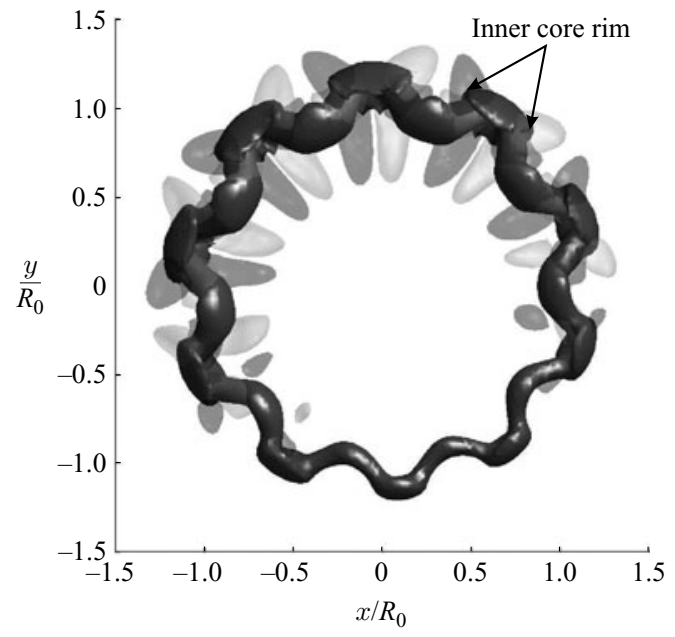

(b)

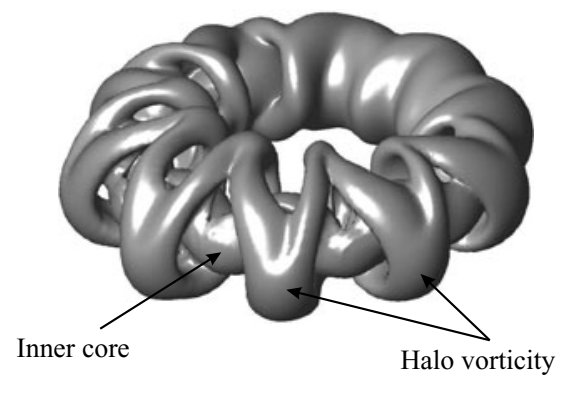

FIgURE 10. Three-dimensional isosurface visualizations of the secondary structures for Case B3 at $\left(t-t^{*}\right) \Gamma_{0} / R_{0}^{2}=65$. (a) Isosurfaces of vorticity viewed from above. Dark surface corresponds to the inner core region $|\omega| R_{0}^{2} / \Gamma=3.8$; mid-grey isosurface corresponds to $\omega_{z} R_{0}^{2} / \Gamma=0.8$, light grey to $\omega_{z} R_{0}^{2} / \Gamma=-0.8$ visualizing the secondary structure. (b) Isosurface of the second invariant of the velocity gradient tensor II, II $R_{0}^{4} / \Gamma^{2}=-0.005$.

with the maximum and minimum inner-core displacements, but displaced radially in opposition to the core displacement. Two loops wrap around each azimuthal wave, hence there are the same number of pairs of loops as there are waves around the ring. Bergdorf et al. (2007) give a slightly different account for the generation of the secondary structure, suggesting they originate because of stretching of the outer-rim regions of the inner core. We also see stretching of the rims of the inner core, but this occurs after the halo vorticity has reorganized into the secondary loops and their magnitude of vorticity $|\omega|$ has become comparable to the inner core.

The generation of the organized secondary structures coincides with deformation of the core $\omega_{\theta}$ distribution. During the laminar regime, the three-dimensional geometry of the core approximates a ring with a mildly elliptic cross-section. During transition the cross-section is distorted to form a thin crescent shape (figure 11). The distortion is more severe in regions of the core in the periphery of the entrainment bubble as the local radial expansion is restricted by the instantaneous zero streamline. The stretching of the inner core intensifies the local vorticity and is accompanied by stretching of the secondary structure, which also intensifies in vorticity causing an overall enstrophy peak (figure $2 b$ ). As the secondary loops stretch they begin to protrude locally outside the entrainment bubble, trailing behind the vortex ring and into the wake. The loops originally developed as counter rotating pairs side by side, but as they trail outside the ring the loops detach and reattach with their neighbour at the saddle point to form hairpin vortices that fill the wake (figure 12), as reported by Bergdorf et al. (2007). The localized equilibrium between the inner core and the outer halo vorticity is broken, as portions of the halo vorticity leave the entrainment bubble, and the core becomes locally turbulent at the position of the initial hairpin vortex shedding. The azimuthal instability wave does not rotate prior to the ring becoming turbulent, which conflicts with the interpretation of Maxworthy (1977). The waves continue to develop across the remainder of the ring unhindered, until the secondary structure is 




FIGURE 11. Contours of $\omega_{\theta}$ on the vertical plane through the ring axis $(y=0)$, for Case A1 at time $\left(t-t^{*}\right) \Gamma_{0} / R_{0}^{2}=116$. Contour increments at $\omega_{\theta \max } / 10$.

(a)



(b)

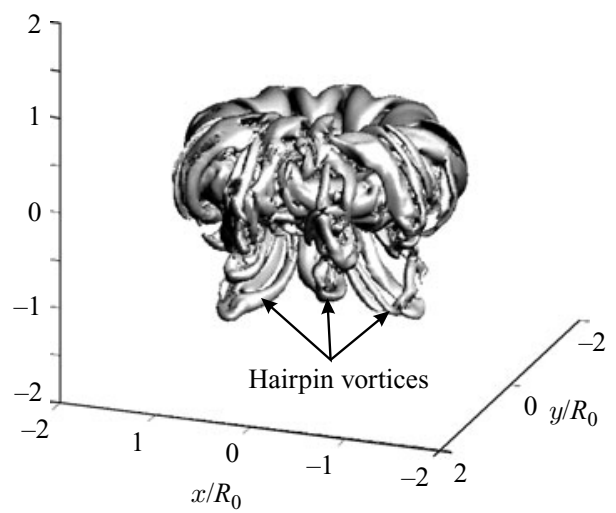

FIGURE 12. Isosurfaces of II showing the termination of the secondary structures forming hairpin vortices in the wake for Case B3 at $(a)\left(t-t^{*}\right) \Gamma_{0} / R_{0}^{2}=75.8$ and $(b)\left(t-t^{*}\right) \Gamma_{0} / R_{0}^{2}=$ 79.4. Surface level II $R_{0}^{4} / \Gamma_{0}^{2}=-0.25$.

shed into hairpin vortices around the entire azimuth of the ring and the ring can be considered to be fully turbulent.

The stationary coherent vortical structure found during the laminar and transitional phases is superseded by the swirling of vorticity filaments. Two cases were simulated and resolved through to transition and into the early turbulent regime: Case A1, a thick-core of low Reynolds number and Case B3, a thin-core of moderate Reynolds number. Figure 13 $(a)$ shows that the thick-core ring breaks down into a number of interwoven vortex filaments. No well-defined coherent core persists and circulation is shed via a continual stream of vortex filaments into the wake. The thin-core ring, however, maintains a core region of concentrated vorticity (the dark region in figure 13b) which is consistent with the turbulent visualizations of Wiegand \& 


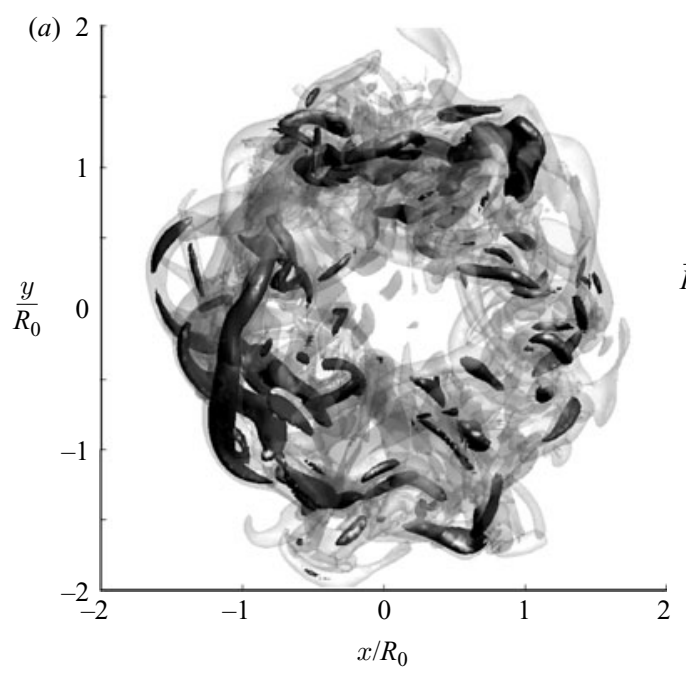

(b)

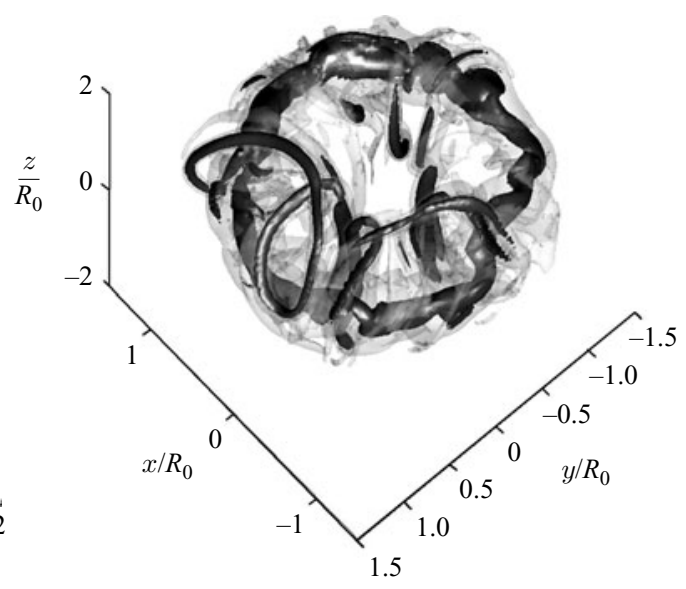

FIGURE 13. Double isosurface of $|\omega|$ for turbulent vortex rings. (a) Case A1 at time $\left(t-t^{*}\right) \Gamma_{0} / R_{0}^{2}=180$ : dark surface level $|\omega| R_{0}^{2} / \Gamma_{0}=1.4$; light surface level $|\omega| R_{0}^{2} / \Gamma_{0}=0.7$. (b) Case B3 at time $\left(t-t^{*}\right) \Gamma_{0} / R_{0}^{2}=111.8$ : dark surface level $|\omega| R_{0}^{2} / \Gamma_{0}=2.5$; light surface level $|\omega| R_{0}^{2} / \Gamma_{0}=1.25$.

Gharib (1994). The core region is no longer stationary, but bends and twists with time. Vorticity filaments, similar to the secondary structure, are continually generated, wrapping around the turbulent core and circulating around it. Figure 13(b) shows a number of these vorticity filaments wrapped round the core region. The filaments have long looped tails that trail into the wake and out of the domain. Just as for the thick-core ring, these vorticity filaments circulate around the core and gradually pass out of the vortex bubble and into the wake as a stream of vorticity filaments and hairpin vortices, as visualized by Glezer \& Coles (1990) and Wiegand \& Gharib (1994). The ring was not simulated further into the turbulent regime; however, the beginning of a staircase-like decay of circulation, as reported by Wiegand \& Gharib (1994) and Bergdorf et al. (2007), was noted.

\subsection{Fluid entrainment/detrainment}

In this section, we analyse the Lagrangian paths of fluid particles in the vicinity of the vortex ring in order to determine its entrainment and detrainment characteristics. The particle paths were obtained by integrating $\mathrm{d} \boldsymbol{x}(t) / \mathrm{d} t=\boldsymbol{v}[\boldsymbol{x}(t), t]$ with a fourthorder Runge-Kutta method, where $\boldsymbol{x}(t)$ is the position vector of the particle and $\boldsymbol{v}$ is the time-dependent velocity field taken from the DNS. The sampling interval of the velocity field was determined to ensure convergence of the path lines, ranging between 5 and 20 time steps dependent on the complexity of the flow field.

We first examine entrainment and detrainment characteristics of the thick-core ring, Case A1, during the laminar regime. As shown in $\$ 3.2$, the laminar ring gradually expands through radial growth and core diffusion, and decelerates to accommodate changes in the bubble geometry and loss of circulation. This increases the distance between the front and rear stagnation points and bubble volume. Figure 14 shows the pathlines of particles released on the plane $z / R_{0}=1.7$ along the circumference of two semi-circles (centred on the ring propagation axis) of radii $0.25 R_{0}$ and $0.35 R_{0}$. The particles initiated on the inner semicircle are entrained whereas the outer ones 


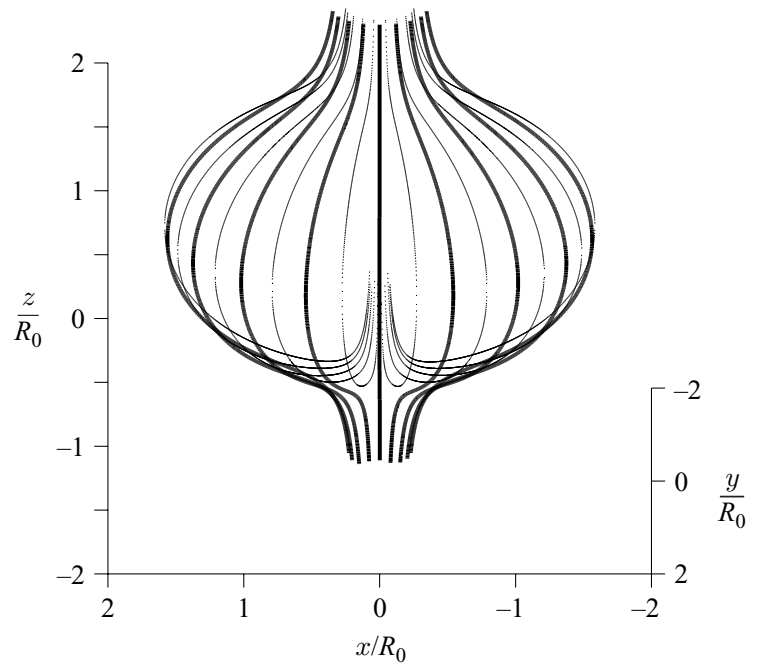

FIGURE 14. Laminar entrainment of tracked particles released at $\left(t-t^{*}\right) \Gamma_{0} / R_{0}^{2}=20$, for Case $\mathrm{A} 1$, on the $z=1.7 R_{0}$ plane. Particles released at equidistant azimuthal locations around the circumference of two semicircles of radius $0.25 R_{0}$ (dotted), and $0.35 R_{0}$ (solid).

pass into the wake, implying a Lagrangian streamtube in front of the vortex ring which separates entrained particles from the external flow. The growth of the bubble is accompanied by molecular diffusion of the azimuthal vorticity $\omega_{\theta}$ across the boundary into the external free stream. There is no detrainment of particles prior to the development of significant secondary structure, and the wake is created solely by vorticity diffusion. In contrast, the Lagrangian bubble interface identified by a Lagrangian coherent structure (LCS) technique in the experiments of Shadden et al. (2006) exhibits alternating lobes of entrainment and detrainment during the laminar regime. Kumar et al. (1995) showed that experimentally generated rings at a similar Reynolds number to those studied by Shadden et al. (2006) initially experience large-scale oscillations in their translational velocity that persist for a short time after formation. We postulate that these experimental rings may also undergo an equilibration phase similar to that observed in our simulation, during which the core adjusts to its steady-state profile. Changes in the distribution of vorticity within the core are sufficient to explain the initial oscillations in the translational velocity and associated lobe dynamics. It was necessary for Shadden et al. to use long integration times when constructing the LCS to reveal the ring's Lagrangian structure. This included part of the time immediately after the ring formation, thus the initial transient is likely to be captured within their results, leading to their observed lobe dynamics.

The laminar entrainment process, characterized by an axisymmetric Lagrangian surface, is significantly altered by the development of the azimuthal instability and associated secondary vortical structure. The first effect of the secondary structure is to deform the Lagrangian surface that defines the entrainment bubble into a wavy interface, which follows the shape of the secondary structure as shown in figure $15(a)$. The bubble deformation also changes the structure of the wake from a circular to a 'petal-like' cross-section at fixed $z$, with the individual petals corresponding to the azimuthal location of the peripheral secondary structure (figure 15b). This petal-like wake structure has been visualized in the experiments of Dazin et al. (2006a). As 
(a)

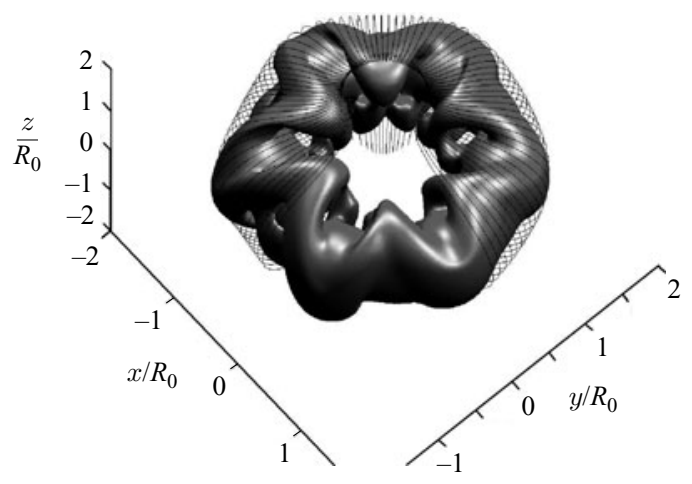

(b)

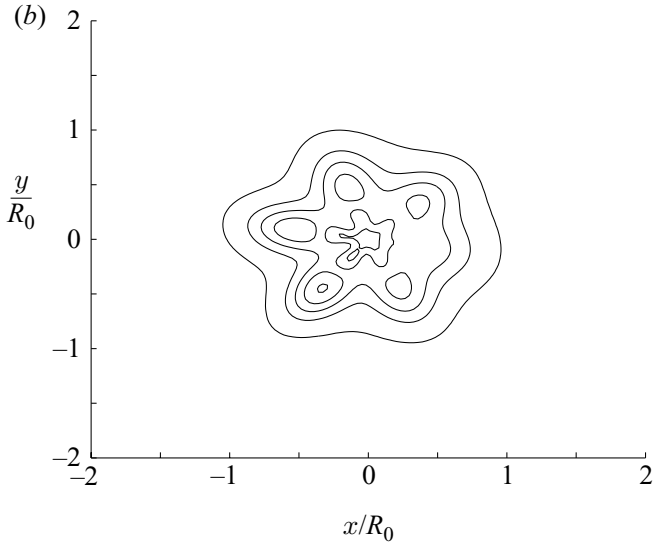

FIGURE 15. Entrainment bubble distortion for Case A1: (a) Lagrangian pathlines of particles initiated around a circle of radius $0.43 R_{0}$ on the $z / R_{0}=1.9$ plane released at $\left(t-t^{*}\right) \Gamma_{0} / R_{0}^{2}=79$. An isosurface of II with surface level II $R_{0}^{4} / \Gamma_{0}^{2}=-0.005$, at $\left(t-t^{*}\right) \Gamma_{0} / R_{0}^{2}=110$ is included to aid visualization; $(b) \omega_{\theta}$ contours across the wake, increments of local $\omega_{\max } / 5$, at $\left(t-t^{*}\right) \Gamma_{0} / R_{0}^{2}=100$ on the $z=-1.95$ plane.

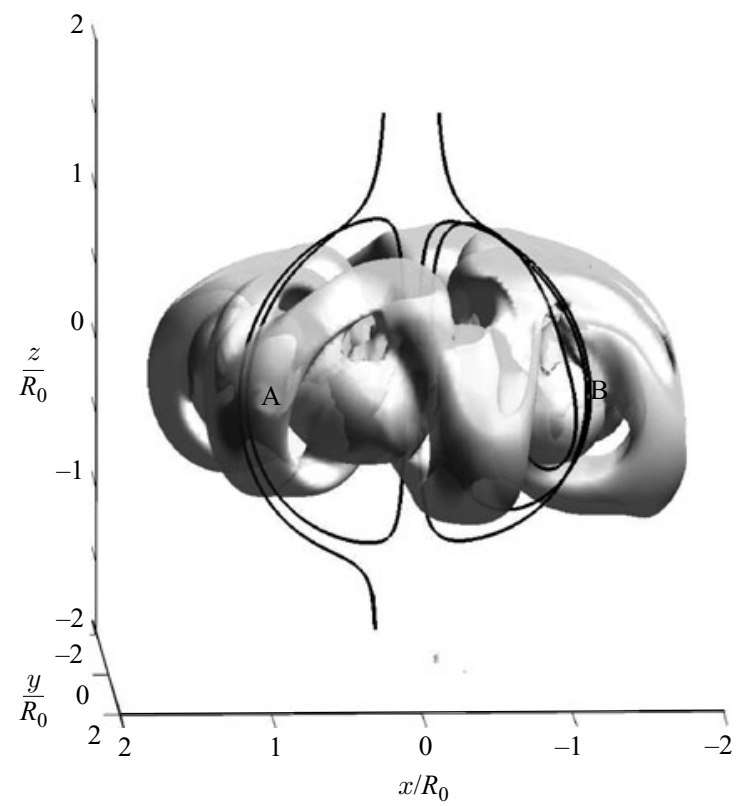

FIGURE 16. Transitional detrainment zones. Two particle pathlines were initiated on the $z / R_{0}=1.9$ plane at $r / R_{0}=0.25$ at time $\left(t-t^{*}\right) \Gamma_{0} / R_{0}^{2}=10$. A translucent isosurface of II with surface level II $R_{0}^{4} / \Gamma_{0}^{2}=-0.005$, at $\left(t-t^{*}\right) \Gamma_{0} / R_{0}^{2}=118$ is included to aid visualization.

the secondary structure develops, the Lagrangian surface is folded to the extent that 'holes' are created through which particles can detrain. The holes are located at points where the secondary loops meet in the outer periphery of the ring. This can be seen by comparing the two particle paths in figure 16, both of which were initiated at the same time and radial distance from the $z$-axis. Just before the onset of turbulence, fluid that is at location A detrains into the wake. Note also the slight drift in the 
(a)

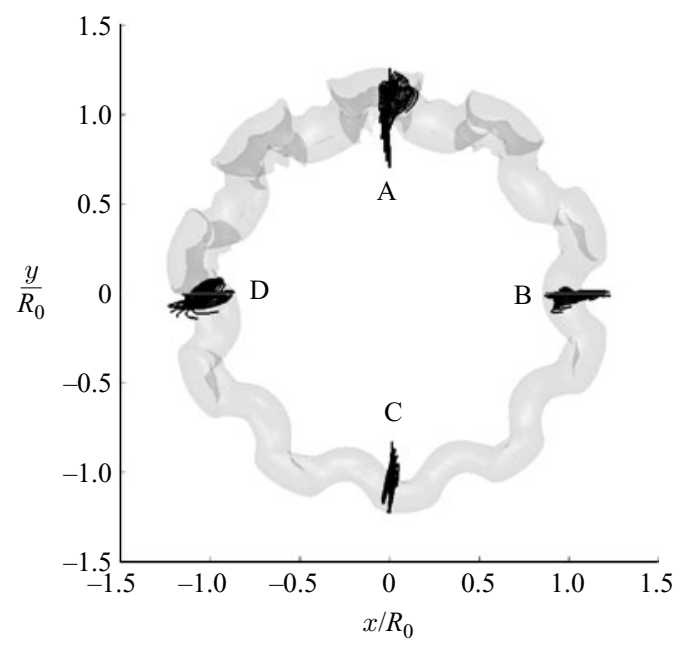

(b)

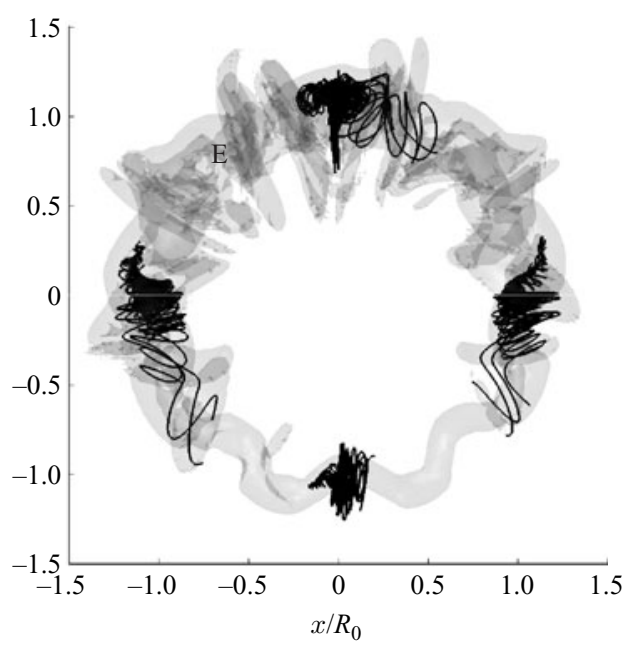

Figure 17. Axial core flow for thin-core ring case B3. (a) Lagrangian pathlines from time $\left(t-t^{*}\right) \Gamma_{0} / R_{0}^{2}=54.2 \rightarrow 60.5$ with translucent isosurface of $|\omega| R_{0}^{2} / \Gamma_{0}=3.0$ at $t=60.5$. (b) Pathlines from time $\left(t-t^{*}\right) \Gamma_{0} / R_{0}^{2}=54.2 \rightarrow 73.1$ with translucent isosurface of $|\omega| R_{0}^{2} /$ $\Gamma_{0}=3.0$ at $\left(t-t^{*}\right) \Gamma_{0} / R_{0}^{2}=73.1$.

left-hand-side particle around the azimuth of the ring. On the other hand, fluid that passes between the loops of the secondary structure, location B, continues to circulate within the entrainment bubble throughout the period of transition. After the onset of turbulence, the ring continually sheds loops of vortical structure, carrying fluid (such as the right-hand-side particle) into the wake. This is the principle mechanism for turbulent detrainment.

\subsection{Axial flow}

We investigate the behaviour of the core region for the possibility of an axial flow being generated prior to the onset of turbulence. Recall that like Maxworthy (1977), we use the term axial flow to refer to circumferential flow along the axis of the vortex core.

The axial flow is analysed by calculating the Lagrangian paths of a number of fluid particles that are initiated within the core along radial lines that intersect the vortex centre at four azimuthal positions, labelled A to D in figure 17(a). The core centre was interpolated from the local velocity field and the Lagrangian pathlines were integrated from the start of the transitional phase to the onset of turbulence.

The thick-core ring (Case A1) shows negligible axial flow. Prior to the onset of turbulence, the particles orbit the core centre and drift through an angle of less than $1^{\circ}$ along the circumferential axis of the core. However, the thin-core ring (Case B3) shows a pronounced axial flow. Figures $17(a)$ and $17(b)$ present the particle paths during the early $\left(54.2 \leqslant\left(t-t^{*}\right) \Gamma_{0} / R_{0}^{2} \leqslant 60.5\right)$ and entire $\left(54.2 \leqslant\left(t-t^{*}\right) \Gamma_{0} / R_{0}^{2} \leqslant 73.1\right)$ transitional phase. During the laminar phase, the particles simply orbit around the centre of the vortex core. However, during the transitional phase, the orbits begin to drift around the ring. The direction in which the particles drift depends on how close they are to the centre of the core. Constructive interference between the azimuthal modes causes a region of increased core stretching, labelled $\mathrm{E}$ in figure 17(b). The individual pathlines indicate an inner region of axial flow directed toward $\mathrm{E}$ (such 
that inner particles at A and B move anticlockwise and C and D move clockwise) and an outer region moving in the opposing direction. The axial flow in the inner-core region is greatest at $\mathrm{B}$, with particles translating $17^{\circ}$ along the circumferential axis of the core during the transitional period. The radius of the inner-core region is approximately $0.078 R_{0}\left(27 \%\right.$ of $\delta_{\theta}$ at time $\left.\left(t-t^{*}\right) \Gamma_{0} / R_{0}^{2}=54.2\right)$. The inner core axial flow is maximum at the core centre and grows approximately exponentially with time.

This type of axial flow, showing a general drift of particles near the core centre toward point $\mathrm{E}$ of strongest instability development, is consistent with the $m=1$ type axial flow recorded by Naitoh et al. (2002), in observations of rings generated through a circular orifice. Naitoh et al. inferred that the axial flow was due to an azimuthal pressure gradient caused by preferential wave growth and associated core stretching. This is supported by our simulations as the thick-core ring undergoes near uniform wave growth leading to negligible axial flow and the thin-core ring experiences preferential wave growth leading to a pronounced axial flow. The thincore ring would be expected to develop with less uniformity as a greater number of modes are excited by the linear instability leading to a larger $n=1$ mode.

\section{Summary and closing remarks}

One important finding is the difference in evolution of thin- and thick-core vortex rings. Analysis of integral measures of vortex-ring geometry has shown that the slenderness ratio $(\epsilon=\delta / R)$ has a strong influence on the evolution of the vortexring geometry and dynamics. The diffusion of thin-core rings is described well by Saffman's equation (2.6) (Saffman 1970). However, the core diffusion of thick-core rings $(\epsilon>0.36)$ is limited by the ring centreline and the presence of the entrainment bubble surface, which clips the outer edge of the vorticity distribution. It was found that as $\epsilon$ increases, the vorticity distribution within the core becomes increasingly skewed with a steepening of the vorticity profile in the vicinity of the entrainment bubble surface and greater vorticity diffusion into a laminar wake. The skewing of the vorticity distribution also has a strong effect on the ring translational velocity, leading us to define an expression for the constant in the classical velocity equation (2.1) as a series expansion of $\epsilon$.

The simulations have shown that thick-cored laminar rings produce a substantial wake, owing to the initial adjustment to a non-Gaussian vorticity distribution and as a result of vorticity detrainment. This has implications for periodic computational domains, used by Shariff et al. (1994) to establish a viscous correction to the inviscid growth rate of Widnall \& Tsai (1977), as we have shown that the interaction between the ring and its recycled wake increases the growth rate of the linear instability. We have presented new revised growth rates from simulations using inflow and outflow boundary conditions in the axis of ring propagation. By modifying the viscous correction to use instantaneous values of the ring parameters we find close agreement for the growth rates of both thin- and thick-core rings.

Visualizing the vortex-ring transition to turbulence has highlighted the importance of the secondary vortical structure, which develops when the inner core instability waves are of large enough amplitude. The secondary structure is formed through tilting and stretching of the outer or 'halo' core vorticity. It develops as counterrotating neighbouring loops of vorticity that meet one another at saddle points to form an interwoven mesh around the inner-core region. It was shown, by analysing Lagrangian pathlines of fluid particles entrained into the vortex ring bubble, that the secondary structure initiates localized detrainment zones prior to the onset of 
turbulence. The latter stages of transition are marked by the protrusion of the secondary structure outside the entrainment bubble, causing it to trail behind into the wake. The neighbouring loops detach and reattach with their neighbour to form hairpin vortices that are deposited into the wake. The local equilibrium between the inner core and the halo vorticity is broken and the ring becomes locally turbulent where the hairpin vortices were ejected. Lagrangian particle analysis of the core region during the transitional phase has shown the generation of an inner region of axial flow which moves along the vortex core centreline toward the region of greatest core stretching. The outer region moves in the opposing direction consistent with the experimental observations of Naitoh et al. (2002) for naturally evolved rings. The dominance of the $n=1$ mode during transition was found to influence the magnitude of the axial flow. The structure of the resultant turbulent ring was found to depend on $\epsilon$ with thin-core rings maintaining a core region of organized vorticity. During the turbulent phase, discrete vortical structure is shed from the ring in the form of hairpin vortices.

This research was funded jointly by the UK Engineering and Physical Sciences Research Council (EPSRC) and the Defence Science and Technology Laboratory (Grant GR/S82299/01) with Dr R.P. Hornby as contract monitor. The work was done as part of the UK Turbulence Consortium, using the facilities of HPCx, the UK's national high-performance computing service, which is provided by EPCC at the University of Edinburgh and by CCLRC Daresbury Laboratory, and funded by the Office of Science and Technology through EPSRC's High End Computing Programme. Special thanks go to Dr C. P. Yorke, Dr J. A. Redford, and Dr K. Shariff for their support, encouragement and expertise.

\section{REFERENCES}

Bergdorf, M., Koumoutsakos, P. \& LeOnard, A. 2007 Direct numerical simulation of vortex rings at $R e_{\gamma}=7500$. J. Fluid Mech. 581, 495-505.

CRow, S. 1970 Stability theory for a pair of trailing vortices. AAIA J. 8, 2172-2179.

Dabiri, J. \& Gharib, M. 2004 Fluid entrainment by isolated vortex rings. J. Fluid Mech. 511, $311-331$.

Dazin, A., Dupont, P. \& Stanislas, M. $2006 a$ Experimental characterization of the instability of the vortex ring. Part I: Linear phase. Exps. Fluids 40, 383-399.

Dazin, A., Dupont, P. \& Stanislas, M. $2006 b$ Experimental characterization of the instability of the vortex ring. Part II: Non-linear phase. Exps. Fluids 41, 401-413.

Glezer, A. \& Coles, D. 1990 An experimental study of a turbulent vortex ring. J. Fluid Mech. 211, 243-283.

Kerswell, R. 2002 Elliptical instability. Annu. Rev. Fluid Mech. 34, 83-113.

KRUTZSCH, C. 1939 Über eine experimentell beobachtete erscheining an werbelringen bei ehrer translatorischen beivegung in weklechin, flussigheiter. Annln Phys. 5, 497-523.

Kumar, M., Arakeri, J. \& Shankar, P. 1995 Translational velocity oscillations of piston generated vortex rings. Phys. Fluids 7, 2751-2756.

Lamb, H. 1932 Hydrodynamics, 6th edn. Dover.

Maxworthy, T. 1972 The structure and stability of vortex rings. J. Fluid Mech. 51, 15-32.

Maxworthy, T. 1974 Turbulent vortex rings. J. Fluid Mech. 64, 227-239.

Maxworthy, T. 1977 Some experimental studies of vortex rings. J. Fluid Mech. 81, 465-495.

Naitoh, T., Fukuda, N., Gotoh, T., Yamada, H. \& Nakajima, K. 2002 Experimental study of axial flow in a vortex ring. Phys. Fluids 14, 143-149.

Rom-Kedar, V., LeOnARD, A. \& Wiggins, S. 1990 An analytical study of transport, mixing and chaos in an unsteady vortical flow. J. Fluid Mech. 214, 347-394.

Saffman, P. 1970 The velocity of viscous vortex rings. Stud. Appl. Maths 49, 371-380. 
Saffman, P. 1978 The number of waves on unstable vortex rings. J. Fluid Mech. 84, 625-639.

SCHNEIDER, P. 1980 Sekundärwirbelbildung bei ringwirbeln und in freistrahlen. Z. Flugwiss. Weltraumforschung 4, 307-318.

Shadden, S., Dabiri, J. \& Marsden, J. 2006 Lagrangian analysis of fluid transport in empirical vortex ring flows. Phys. Fluids 18, 047105.

Shariff, K., Verzicco, R. \& Orlandi, P. 1994 A numerical study of three-dimensional vortex ring instabilities: viscous corrections and early nonlinear stage. J. Fluid Mech. 279, 351-375.

Shariff, K., Leonard, A. \& Ferziger, J. 2006 Dynamical systems analysis of fluid transport in time-periodic vortex ring flows. Phys. Fluids 18, 047104.

Widnall, S. 1975 The structure and dynamics of vortex filaments. Annu. Rev. Fluid Mech. 7, 141-165.

Widnall, S. \& Sullivan, J. 1973 On the stability of vortex rings. Proc. R. Soc. Lond. A 332, 335-353.

Widnall, S. \& TsaI, C.-Y. 1977 The instability of the thin vortex ring of constant vorticity. Phil. Trans. R. Soc. Lond. 287, 273-305.

Wiegand, A. \& Gharib, M. 1994 On the decay of a turbulent vortex ring. Phys. Fluids 38, 3806-3808.

Yao, Y., Thomas, T., Sandham, N. \& Williams, J. 2001 Direct numerical simulation of turbulent flow over a rectangular trailing edge. Theoret. Comput. Fluid Dyn. 14, 337-358. 